\title{
Pattern rigidity and the Hilbert-Smith conjecture
}

\author{
MAHAN MJ
}

We initiate a study of the topological group $\operatorname{PPQI}(G, H)$ of pattern-preserving quasiisometries for $G$ a hyperbolic Poincaré duality group and $H$ an infinite quasiconvex subgroup of infinite index in $G$. Suppose $\partial G$ admits a visual metric $d$ with $\operatorname{dim}_{\text {haus }}<$ $\operatorname{dim}_{t}+2$, where $\operatorname{dim}_{\text {haus }}$ is the Hausdorff dimension and $\operatorname{dim}_{t}$ is the topological dimension of $(\partial G, d)$. Equivalently suppose that $\operatorname{ACD}(\partial G)<\operatorname{dim}_{t}+2$, where $\operatorname{ACD}(\partial G)$ denotes the Ahlfors regular conformal dimension of $\partial G$.

(a) If $Q_{u}$ is a group of pattern-preserving uniform quasi-isometries (or more generally any locally compact group of pattern-preserving quasi-isometries) containing $G$, then $G$ is of finite index in $Q_{u}$.

(b) If instead, $H$ is a codimension one filling subgroup, and $Q$ is any group of pattern-preserving quasi-isometries containing $G$, then $G$ is of finite index in $Q$. Moreover, if $L$ is the limit set of $H, \mathcal{L}$ is the collection of translates of $L$ under $G$, and $Q$ is any pattern-preserving group of homeomorphisms of $\partial G$ preserving $\mathcal{L}$ and containing $G$, then the index of $G$ in $Q$ is finite (Topological Pattern Rigidity).

We find analogous results in the realm of relative hyperbolicity, regarding an equivariant collection of horoballs as a symmetric pattern in the universal cover of a complete finite volume noncompact manifold of pinched negative curvature. Our main result combined with a theorem of Mosher, Sageev and Whyte gives QI rigidity results.

An important ingredient of the proof is a version of the Hilbert-Smith conjecture for certain metric measure spaces, which uses the full strength of Yang's theorem on actions of the p-adic integers on homology manifolds. This might be of independent interest.

20F67; 57M50, 22E40

\section{Preliminaries}

\subsection{Statement of results}

In this paper we start studying the full group of "pattern-preserving quasi-isometries" for pairs $(G, H)$, where $G$ is a (Gromov) hyperbolic group and $H$ an infinite quasiconvex 
subgroup of infinite index in $G$. Gromov [31] proposed the project of classifying finitely generated groups up to quasi-isometry, as well as the study of the group $\mathrm{QI}(X)$ of quasi-isometries of a space $X$, where two quasi-isometries are identified if they lie at a bounded distance from each other. A class of groups where any two members are quasiisometric if and only if they are commensurable is said to be quasi-isometrically rigid. However, any class of groups acting freely, cocompactly and properly discontinuously on some fixed proper hyperbolic metric space $\mathbf{H}$ are quasi-isometric to $\mathbf{H}$ and hence to each other. In this context (or in a context where quasi-isometric rigidity is not known) it makes sense to ask a relative version of Gromov's question. To obtain rigidity results, we impose additional restrictions on the quasi-isometries by requiring that they preserve some additional structure given by a "symmetric pattern" of subsets. A "symmetric pattern" of subsets roughly means a $G$-equivariant collection $\mathcal{J}$ of convex (or uniformly quasiconvex) cocompact subsets in $\mathbf{H}$ (see Section 1.3 for detailed definitions). Then the relative version of Gromov's question for classes of pairs $(G, H)$ was formulated by Mosher, Sageev and Whyte [46] as the following pattern rigidity question:

Question 1.1 Given a quasi-isometry $q$ of two such pairs $\left(G_{i}, H_{i}\right)(i=1,2)$ pairing a $\left(G_{1}, H_{1}\right)$-symmetric pattern $\mathcal{J}_{1}$ with a $\left(G_{2}, H_{2}\right)$-symmetric pattern $\mathcal{J}_{2}$, does there exist an abstract commensurator $I$ which performs the same pairing?

The study of this question was initiated by Schwartz [56; 57], where $G$ is a lattice in a rank one symmetric space. The paper [56] deals with symmetric patterns of convex sets (horoballs) whose limit sets are single points, and [57] deals with symmetric patterns of convex sets (geodesics) whose limit sets consist of two points. Biswas and $\mathrm{Mj}$ [7] generalized Schwartz' result to certain Duality and PD subgroups of rank one symmetric spaces. Biswas [6] completely solved the pattern rigidity problem for $G$ a uniform lattice in real hyperbolic space and $H$ any infinite quasiconvex subgroup of infinite index in $G$. However, all these papers used, in an essential way, the linear structure of the groups involved, and the techniques fail for $G$ the fundamental group of a general closed negatively curved manifold. (This point is specifically mentioned by Schwartz in [57]). Further, the study in $[56 ; 57 ; 7 ; 6]$ boils down to the study of a single pattern-preserving quasi-isometry between pairs $\left(G_{1}, H_{1}\right)$ and $\left(G_{2}, H_{2}\right)$. We propose a different perspective in this paper by studying the full group $\operatorname{PPQI}(G, H)$ of pattern-preserving (self-)quasi-isometries of a pair $(G, H)$ for $G$ a hyperbolic group and $H$ any infinite quasiconvex subgroup of infinite index. The features of $G$ that we shall use are general enough to go beyond the linear context while at the same time being strong enough to ensure rigidity in certain contexts. Some of the ingredients of this paper are: 
(1) The boundary of a Poincare duality (PD for short) hyperbolic group is a homology manifold (cf Definition 1.14) by a Theorem of Bestvina and Mess [4].

(2) The algebraic topology of homology manifolds imposes restrictions on what kinds of groups may act on them by Theorems of Newman [48], Smith [59] and Yang [67].

(3) Boundaries of hyperbolic groups equipped with the visual metric also have a metric measure space structure with the property that they are Ahlfors regular (cf Definition 2.10).

(4) Quasiconformal analysis can be conducted in the general context of Ahlfors regular metric spaces.

(5) A combinatorial cross-ratio can be constructed on the boundary of a hyperbolic group in the presence of a codimension one subgroup. (Roughly speaking these are subgroups whose Cayley graphs coarsely separate the Cayley graph of the group. See first paragraph of Section 4.1 for a formal definition of codimension one subgroups.)

We refer the reader to Hocking and Young [37, page 145] for details on topological dimension and Davis [23] for details on PD groups.

Of these ingredients, the first two come from (a somewhat forgotten chapter of) algebraic topology, the next two from a very active new area of analysis on metric measure spaces, while the last comes from geometric group theory proper. Topological actions of finite groups on manifolds and homological consequences of actions of $p$-adics on manifolds form the two main ingredients for a proof of the Hilbert-Smith conjecture for bi-Lipschitz (see Repovš and S̆čepin [54]) and quasiconformal (see Martin [42]) actions. We first generalize the result of Martin [42] to Ahlfors regular metric spaces that are boundaries of PD hyperbolic groups and obtain the following.

Theorem 2.24 and Corollary 2.25 Let $G$ be a Poincare duality hyperbolic group and $Q$ be a group of (boundary values of) quasi-isometries of $G$. Suppose $d$ is a visual metric on $\partial G$ with $\operatorname{dim}_{\text {haus }}<\operatorname{dim}_{t}+2$, where $\operatorname{dim}_{\text {haus }}$ is the Hausdorff dimension (cf Definition 2.9) and $\operatorname{dim}_{t}$ is the topological dimension of $(\partial G, d)$. Equivalently suppose that $\operatorname{ACD}(\partial G)<\operatorname{dim}_{t}+2$, where $\operatorname{ACD}(\partial G)$ denotes the Ahlfors regular conformal dimension (cf Definition 2.13) of $\partial G$. Then $Q$ cannot contain a copy of $Z_{(p)}$, where $Z_{(p)}$ denotes the $p$-adic integers. Hence if $Q$ is finite dimensional locally compact, it must be a Lie group.

Theorem 2.24 and Corollary 2.25 give a strong affirmative answer to a question of Iwaniec and Martin [38, Remark 2, page 527]. 
As in [42], there is no assumption on the uniformity of the quasiconformal maps in $Z_{(p)}$. The analogue of Theorem 2.24 is false for purely topological actions (see Raymond and Williams [53]) on homology manifolds. Hence the quasi-isometry assumption is crucial here. The statement that a topological manifold does not admit an effective topological $Z_{(p)}$ action is the famous Hilbert-Smith conjecture (but does not imply Corollary 2.25).

Corollary 2.25 will be a crucial ingredient in our approach to pattern rigidity. Another property we shall be investigating in some detail is the notion of "topological infinite divisibility" (see Section 3.1 for definitions). The notion we introduce is somewhat weaker than related existing notions in the literature. In this generality, we prove:

Propositions 3.5 and 3.7 Let $G$ be a hyperbolic group and $H$ an infinite quasiconvex subgroup of infinite index in $G$.

(a) Any group of pattern-preserving quasi-isometries is totally disconnected and contains no topologically infinitely divisible elements.

(b) If $G$ is a Poincare duality group, the group $\mathrm{QI}(G)$ of quasi-isometries cannot contain arbitrarily small torsion elements.

We obtain stronger results under the assumption that $G$ is a Poincare duality group (eg the fundamental group of a closed negatively curved manifold) with some restrictions on the visual metric on its boundary. (We refer the reader to the first paragraph of Section 4.1 for the notion of codimension one filling subgroups.)

Theorems 3.9, 4.8 and 4.10 Let $G$ be a hyperbolic Poincare duality group and $H$ an infinite quasiconvex subgroup of infinite index in $G$. Suppose further that for some visual metric on $\partial G$, $\operatorname{dim}_{\text {haus }}(\partial G)<\operatorname{dim}_{t}(\partial G)+2$, where $\operatorname{dim}_{\text {haus }}$ and $\operatorname{dim}_{t}$ denote Hausdorff and topological dimension respectively. Equivalently suppose that $\operatorname{ACD}(\partial G)<\operatorname{dim}_{t}+2$, where $\operatorname{ACD}(\partial G)$ denotes the Ahlfors regular conformal dimension of $\partial G$.

(a) If $Q_{u}$ is a group of pattern-preserving uniform quasi-isometries (or more generally any locally compact group of pattern-preserving quasi-isometries) containing $G$, then $G$ is of finite index in $Q_{u}$.

(b) If further, $H$ is a codimension one filling subgroup, and $Q$ is any group of (not necessarily uniform) pattern-preserving quasi-isometries containing $G$, then $G$ is of finite index in $Q$.

(c) (Topological Pattern Rigidity) Under the assumptions of (b), let $L$ be the limit set of $H$ and $\mathcal{L}$ be the collection of translates of $L$ under $G$. Let $Q$ be any pattern-preserving group of homeomorphisms of $\partial G$ preserving $\mathcal{L}$ and containing $G$. Then the index of $G$ in $Q$ is finite. 
Theorem 4.10 is a generalization of a Theorem of Casson and Bleiler [19] and Kapovich and Kleiner [39] to all dimensions. Casson and Bleiler [19] and Kapovich and Kleiner [39] proved Theorem 4.10 for $G$ the fundamental group of a surface and $H$ an infinite cyclic subgroup corresponding to a filling curve.

Codimension one filling subgroups (See first paragraph of Section 4.1 for definitions.) The existence of a codimension one filling quasiconvex subgroup $H$ (or more generally a finite family of codimension one quasiconvex subgroups that are filling as a collection) of a hyperbolic group $G$ ensures that $G$ acts properly, cocompactly on a CAT (0) cube complex (see Sageev [55]) by a recent result of Bergeron and Wise [2]. Thus, Theorems 3.9, 4.8 and 4.10 deal with the pattern-rigidity for $\operatorname{PD}(n)$ hyperbolic groups acting properly and cocompactly on a CAT( 0$)$ cube complex.

We also derive QI rigidity results for fundamental groups of certain noncompact negatively curved manifolds of finite volume, by deriving analogues of Theorem 3.9 for symmetric patterns of horoballs and combining it with a Theorem of Behrstock, Druţu and Mosher [1]. The hypotheses in the following Theorem are satisfied by fundamental groups of finite volume complete noncompact manifolds of sufficiently pinched negative curvature and dimension bigger than 2 .

Theorem 5.5 Let $M=M^{n}$ be a complete finite volume manifold of pinched negative curvature with $n>2$. Let $G=\pi_{1}(M)$. Suppose that there exists a visual metric $d$ on $\partial(\widetilde{M})$ with $\operatorname{dim}_{\text {haus }}<\operatorname{dim}_{t}+2$, where $\operatorname{dim}_{\text {haus }}$ is the Hausdorff dimension and $\operatorname{dim}_{t}$ is the topological dimension of $(\partial(\widetilde{M}), d)$. Equivalently suppose that $\operatorname{ACD}(\partial(\widetilde{M}))<$ $\operatorname{dim}_{t}+2$, where $\operatorname{ACD}(\partial(\widetilde{M}))$ denotes the Ahlfors regular conformal dimension of $\partial(\widetilde{M})$.

Let $\Gamma$ be a Cayley graph of $G$ with respect to a finite generating set. Let $Q$ be a group of uniform quasi-isometries of $\Gamma$ containing $G$. Then $G$ is of finite index in $Q$. In particular, $Q \subset \operatorname{Comm}(G)$, where $\operatorname{Comm}(G)$ denotes the abstract commensurator of $G$.

The author learnt the following Scholium from Misha Gromov [32].

Scholium 1.2 If two discrete groups can be embedded in the same locally compact group nicely, they are as good as commensurable.

A partial aim of this paper is to make Scholium 1.2 precise in the context of pattern rigidity. It follows from Theorems 3.9 and 5.5 that in the context of pattern rigidity or QI rigidity of (fundamental groups of) finite volume complete noncompact manifolds of 
pinched negative curvature and dimension bigger than 2, "as good as" can be replaced by "actually" in Scholium 1.2. Thus, Theorem 3.9 and Theorem 5.5 reduce the problem of pattern rigidity to the weaker problem of embedding two groups simultaneously in the same locally compact group and Theorem 4.8 carries out this embedding under certain hypotheses.

Notation To prevent confusion we fix two pieces of notation:

(1) $\mathbb{Z}_{p}$ will denote the integers $\bmod p$.

(2) $Z_{(p)}$ will denote the $p$-adic integers.

Section 2 discusses Ahlfors regular metric spaces and proves the Hilbert-Smith conjecture in our context. In Section 3 we investigate pattern preserving groups as topological groups, including topological infinite divisibility and PD groups. Section 4 considers filling codimension one subgroups and pattern rigidity. Section 5 specializes to finite volume manifolds of negative curvature, and in Section 6 we give examples and consequences of our results.

\subsection{Dotted geodesic metric spaces}

Definition 1.3 A dotted metric space is a metric space $X$, where $d(x, y)$ is an integer for all $x, y \in X$. A dotted geodesic metric space is a dotted metric space $X$, such that for all $x, y \in X$, there exists an isometric map $\sigma:[0, d(x, y)] \cap \mathbb{Z} \rightarrow X$ with $\sigma(0)=x$ and $\sigma(d(x, y))=y$. A dotted metric space is proper if every ball $N_{k}(x)$ is finite.

The compact open topology on the space $\mathcal{F}$ of self-maps of a dotted metric space is defined by taking the family of sets $U_{K}(f)=\{g \in \mathcal{F}: g(x)=f(x), \forall x \in K$, $K \subset X$ finite $\}$ as a basis for the topology on $\mathcal{F}$.

The following easy observation will turn out to be quite useful. Note that we do not need any extra geometric assumptions (eg hyperbolicity) on $X$ in the Lemma below.

Lemma 1.4 Let $(X, d)$ be a proper dotted geodesic metric space. Let $L$ be a closed subset of $\mathcal{F}$, the collection of self-maps of $X$ equipped with the compact open topology. Further suppose that there exist $K \geq 1, C, \epsilon \geq 0$ and $x \in X$ such that for all $g \in L$, $g$ is a $(K, \epsilon)$ quasi-isometry of $X$ and $d(x, g(x)) \leq C$. Then $L$ is compact. Hence any group of uniform quasi-isometries of a proper dotted metric space $X$ is locally compact. 
Proof Since $N_{C}(x)$ is finite (by properness), it suffices to prove that for all $y \in N_{C}(x)$, $\{g \in L: g(x)=y\}$ is compact.

Hence, without loss of generality assume that there exists $y \in X$ such that $g(x)=y$ for all $g \in L$. Since $X$ is proper, $X$ is countable. Let $X=\left\{x=x_{1}, x_{2}, \ldots, x_{n}, \ldots\right\}$ be an enumeration of the elements of $X$. Since each $g_{\alpha} \in L$ is a $(K, \epsilon)$-quasi-isometry and $g(x)=y$ for all $g \in L$, then for each $x_{n} \in X$, there exists a finite set $K_{n}$ such that $g\left(x_{n}\right) \in K_{n}$ for all $g \in L$.

Then, given any infinite collection of $g_{\alpha}$ 's in $L$, we can pass to a sequence $\left\{g_{1}, g_{2}, \ldots\right.$, $\left.g_{n}, \ldots\right\}$ such that for each $x_{n} \in X$, there exists $y_{n} \in X$ with $g_{i}\left(x_{n}\right)=y_{n}$ for all $i \leq n$. Let $g_{\infty}\left(x_{n}\right)=y_{n}$ for all $n$.

Then $g_{n}$ converges to $g_{\infty}$ in $\mathcal{F}$, the collection of self-maps of $X$ equipped with the compact open topology and we are done.

Lemma 1.4 may be thought of as a coarsening of the fact that the stabilizer of a point in the isometry group of a Riemannian manifold is compact.

For a nonelementary Gromov hyperbolic group $G$ we shall construct a certain pseudometric space which will come in handy. It is known [30] that $G$ acts cocompactly on the collection $\partial^{3} G$ of distinct triples on the boundary $\partial G$ of $G$. Let $K$ be a (closed) fundamental domain for this action. Choose a point $p$ in the interior $\operatorname{Int}(K)$ of $K$. Define $\rho(g(p), h(p))=1$ if $g(K) \cap h(K) \neq \varnothing$. Also for $x \in g(\operatorname{Int}(K))$ define $\rho(g(p), x)=0$. For $x \in \partial^{3} G \backslash \bigcup_{g} g(\operatorname{Int}(K))$, let $g_{1}, \ldots, g_{m}$ be the collection of all elements of $G$ such that $x \in \bigcap_{i} g_{i}(K)$. Choose one of the elements $g_{1}, \ldots, g_{m}$, say $g_{i}$ and define $\rho\left(g_{i}(p), x\right)=0$ and $\rho\left(g_{j}(p), x\right)=1$ for $j \neq i$. Now define a dotted path metric on $\partial^{3} G$ by $\rho(x, y)=\inf \left\{n:\right.$ there exists a sequence $x=x_{0}, g_{1}(p), g_{2}(p), \ldots, g_{n}(p), x_{n+1}=y$ such that $\rho\left(x_{0}, g_{1}(p)\right)=0=\rho\left(x_{n+1}, g_{n}(p)\right)$ and $\rho\left(g_{i}(p), g_{i+1}(p)\right)=1$ for $\left.i=1, \ldots, n-1\right\}$.

Observation 1.5 The pseudo-metric space $\left(\partial^{3} G, \rho\right)$ is quasi-isometric to any Cayley graph $\Gamma$ of $G$ with respect to a finite generating set. The proof of this fact is an easy modification of the Švarc-Milnor Lemma (see the proof [16, Proposition 8.19, page 140]). The map $\phi: \Gamma \rightarrow\left(\partial^{3} G, \rho\right)$ given by $\phi(g)=g(p)$ gives the required quasi-isometry.

\subsection{Patterns}

Definition 1.6 Let $G$ be a hyperbolic group acting geometrically (ie freely, cocompactly and properly discontinuously by isometries) on a hyperbolic metric space $\mathbf{H}$. 
A symmetric pattern of closed convex (or quasiconvex) sets in $\mathbf{H}$ is a $G$-invariant countable collection $\mathcal{J}$ of convex (or quasiconvex) sets such that

(1) the stabilizer $H$ of $J \in \mathcal{J}$ acts cocompactly on $J$,

(2) $\mathcal{J}$ is the orbit of some (any) $J \in \mathcal{J}$ under $G$.

This definition is slightly more restrictive than Schwartz' notion of a symmetric pattern of geodesics, in the sense that he takes $\mathcal{J}$ to be a finite union of orbits of geodesics, whereas Condition (2) above forces $\mathcal{J}$ to consist of one orbit. All our results go through with the more general definition, where $\mathcal{J}$ is a finite union of orbits of closed convex (or quasiconvex) sets, but we restrict ourselves to one orbit for ease of exposition.

Suppose that $\left(X_{1}, d_{1}\right),\left(X_{2}, d_{2}\right)$ are metric spaces. Let $\mathcal{J}_{1}, \mathcal{J}_{2}$ be collections of closed subsets of $X_{1}, X_{2}$ respectively. Then $d_{i}$ induces a pseudo-metric (which, by abuse of notation, we continue to refer to as $d_{i}$ ) on $\mathcal{J}_{i}$ for $i=1,2$. This is just the ordinary (not Hausdorff) distance between closed subsets of a metric space.

In particular, consider two hyperbolic groups $G_{1}, G_{2}$ with quasiconvex subgroups $H_{1}, H_{2}$, Cayley graphs $\Gamma_{1}, \Gamma_{2}$. Let $\mathcal{L}_{j}$ for $j=1,2$ denote the collection of translates of limit sets of $H_{1}, H_{2}$ in $\partial G_{1}, \partial G_{2}$ respectively. Individual members of the collection $\mathcal{L}_{j}$ will be denoted as $L_{i}^{j}$. Let $\mathcal{J}_{j}$ denote the collection $\left\{J_{i}^{j}=J\left(L_{i}^{j}\right): L_{i}^{j} \in \mathcal{L}_{j}\right\}$ of joins of limit sets. Recall that the join of a limit set $\Lambda_{i}$ is the union of bi-infinite geodesics in $\Gamma_{i}$ with endpoints in $\Lambda_{i}$. This is a uniformly quasiconvex set and lies at a bounded Hausdorff distance from the Cayley graph of the subgroup $H_{i}$ (assuming that the Cayley graph of $H_{i}$ is taken with respect to a finite generating set which is contained in the generating set of $G_{i}$ ). Following Schwartz [57], we define:

Definition 1.7 A bijective map $\phi$ from $\mathcal{J}_{1} \rightarrow \mathcal{J}_{2}$ is said to be uniformly proper if there exists a function $f: \mathbb{N} \rightarrow \mathbb{N}$ such that

$$
\begin{aligned}
& \text { (1) } d_{G_{1}}\left(J\left(L_{i}^{1}\right), J\left(L_{j}^{1}\right)\right) \leq n \Rightarrow d_{G_{2}}\left(\phi\left(J\left(L_{i}^{1}\right)\right), \phi\left(J\left(L_{j}^{1}\right)\right)\right) \leq f(n), \\
& \text { (2) } d_{G_{2}}\left(\phi\left(J\left(L_{i}^{1}\right)\right), \phi\left(J\left(L_{j}^{1}\right)\right)\right) \leq n \Rightarrow d_{G_{1}}\left(J\left(L_{i}^{1}\right), J\left(L_{j}^{1}\right)\right) \leq f(n) .
\end{aligned}
$$

When $\mathcal{J}_{i}$ consists of all singleton subsets of $\Gamma_{1}, \Gamma_{2}$, we shall refer to $\phi$ as a uniformly proper map from $\Gamma_{1}$ to $\Gamma_{2}$.

The proof of the following Theorem can be culled out of our paper [44]. We give a proof for completeness. 
Theorem 1.8 Let $H$ be an infinite quasiconvex subgroup of a hyperbolic group $G$ such that $H$ has infinite index in $G$. Let $\Gamma$ be a Cayley graph of $G$ with metric $d$. Let $L$ be the limit set of $H$ and $\mathcal{L}$ be the collection of translates of $L$ under $G$. There exists a finite collection $L_{1}, \ldots, L_{n}$ of elements of $\mathcal{L}$ such that the following holds.

For any $K, \epsilon$, there exists a $C$ such that if $\phi: \Gamma \rightarrow \Gamma$ is a pattern-preserving $(K, \epsilon)-$ quasi-isometry of $\Gamma$ with $\partial \phi\left(L_{i}\right)=L_{i}$ for $i=1, \ldots, n$, then $d(\phi(1), 1) \leq C$.

Proof Let $J_{i}$ denote $J\left(L_{i}\right)$ for $L_{i} \in \mathcal{L}$. Also let $B_{k}(1)$ denote the $k$-neighborhood of $1 \in \Gamma$. In [44, page 1706], we show that there exists $M \in \mathbb{N}$ such that for all $k \geq M$ the collection

$$
\left\{J_{i}: B_{k}(1) \cap J_{i} \neq \varnothing\right\}
$$

contains a pair $J_{p}, J_{q}$ such that $L_{p} \cap L_{q}=\varnothing$. Further [44, page 1707] for any $K_{1}$, there exists $D$, such that $\left\{z \in \Gamma: d\left(z, J_{p}\right) \leq K_{1}, d\left(z, J_{q}\right) \leq K_{1}\right\}$ has diameter less than $D$.

Let $L_{1}, \ldots L_{n}$ be all the elements of $\mathcal{L}$ such that $B_{M}(1) \cap J_{i} \neq \varnothing$. Suppose $\partial \phi\left(L_{i}\right)=L_{i}$ for $i=1, \ldots, n$. Then there exists $C_{0}=C_{0}(K, \epsilon)$ such that $\phi\left(J_{i}\right)$ lies in a $C_{0}$ neighborhood of $J_{i}$ for $i=1, \ldots, n$. Also $d\left(\phi(1), \phi\left(J_{i}\right)\right) \leq M K+\epsilon$ for $i=1, \ldots, n$. Hence $d\left(\phi(1), J_{i}\right) \leq M K+\epsilon+C_{0}$ for $i=1, \ldots, n$.

Choose $K_{1}=M K+\epsilon+C_{0}$. Then $d\left(z, J_{p}\right) \leq K_{1}, d\left(z, J_{q}\right) \leq K_{1}$ for $z=1$ or $z=\phi(1)$. Hence $d(1, \phi(1)) \leq D$ where $D$ is the real number determined by the last assertion in the first paragraph of this proof. Taking $C=D$ we are done.

Definition 1.9 Let $H$ be an infinite quasiconvex subgroup of a hyperbolic group $G$ such that $H$ has infinite index in $G$. The group $\operatorname{PP}(G, H)$ of pattern-preserving maps for such a pair $(G, H)$ is defined as the group of homeomorphisms of $\partial G$ that preserve the collection of translates $\mathcal{L}$, ie $\operatorname{PP}(G, H)=\{\phi \in \operatorname{Homeo}(\partial G): \phi(L) \in \mathcal{L}, \forall L \in \mathcal{L}\}$. The group $\operatorname{PPQI}(G, H)$ of pattern-preserving quasi-isometries for a pair $(G, H)$ as above is defined as the subgroup of $\operatorname{PP}(G, H)$ consisting of homeomorphisms $h$ of $\partial G$ such that $h=\partial \phi$ for some quasi-isometry $\phi: \Gamma \rightarrow \Gamma$. The topology on $\operatorname{PP}(G, H)$ or $\operatorname{PPQI}(G, H)$ is inherited (as a subspace) from the uniform topology on $\operatorname{Homeo}(\partial G)$.

Proposition 1.10 [44] The collection $\mathcal{L}$ is discrete in the Hausdorff topology on the space of closed subsets of $\partial G$, ie for all $L \in \mathcal{L}$, there exists $\epsilon>0$ such that $N_{\epsilon}(L) \cap \mathcal{L}=L$, where $N_{\epsilon}(L)$ denotes an $\epsilon$ neighborhood of $L$ in the Hausdorff metric. Further, for every $\epsilon>0$ and any visual metric $d$ on $\partial G$, the number of elements of $\mathcal{L}$ of diameter greater than $\epsilon$ is finite. 
Observation 1.11 We observe now that $\operatorname{PP}(G, H)$ is closed in Homeo $(\partial G)$ equipped with the uniform topology. To see this, assume that $f_{n} \in \operatorname{PP}(G, H)$ and $f_{n} \rightarrow f$ in Homeo( $\partial G)$ equipped with the uniform topology. If $f \notin \operatorname{PP}(G, H)$ there exists $L \in \mathcal{L}$ such that $f(L) \notin \mathcal{L}$. Since $f_{n}$ converges to $f$ in the uniform topology, $f_{n}(L) \rightarrow f(L)$ in the Hausdorff metric on closed subsets of $\partial G$. Since $f$ is a homeomorphism, there exists $\epsilon>0$ such that the diameter of $f(L)$ is greater than $2 \epsilon$ and hence there exists $N \in \mathbb{N}$ such that the diameter of $f(L)$ is greater than $\epsilon$ for all $n \geq N$. Since the number of elements of $\mathcal{L}$ of diameter greater than $\epsilon$ is finite by Proposition 1.10, then (after passing to a subsequence if necessary) it follows that there exists $L_{1} \in \mathcal{L}$ such that $f_{n}(L)=L_{1}$ for all $n \geq N$. Since $L, L_{1}$ are closed and $f_{n} \rightarrow f$ in Homeo( $\left.\partial G\right)$ equipped with the uniform topology, it follows that $f(L)=L_{1} \in \mathcal{L}$, a contradiction. This shows that $\operatorname{PP}(G, H)$ is closed in $\operatorname{Homeo}(\partial G)$.

The same is true for $\operatorname{PPQI}(G, H)$.

Henceforth, whenever we refer to $\operatorname{Homeo}(\partial G)$ as a topological group, we shall assume that it is equipped with the uniform topology.

Combining Lemma 1.4 with Theorem 1.8, we get:

Corollary 1.12 Let $H$ be an infinite quasiconvex subgroup of infinite index in a hyperbolic group $G$. Let $\Gamma$ be a Cayley graph of $G$ with metric $d$ and $Q \subset \operatorname{PPQI}(G, H)$ be any group of boundary values of uniform quasi-isometries of (the vertex set of) $\Gamma$. Let $L$ be the limit set of $H$ and $\mathcal{L}$ be the collection of translates of $L$ under $G$. There exists a finite collection $L_{1}, \ldots L_{n}$ of elements of $\mathcal{L}$ such that $Q_{0}=\bigcap_{i=1, \ldots, n} \operatorname{Stab}\left(L_{i}\right)$ is compact, where $\operatorname{Stab}\left(L_{i}\right)$ denotes the stabilizer of $L_{i}$ in $Q$. (Here $Q$ is equipped with the uniform topology as a subspace of $\operatorname{Homeo}(\partial G)$.)

Proof Let $K \geq 1, \epsilon \geq 0$ be such that each $q \in Q$ is the boundary value of a $(K, \epsilon)-$ quasi-isometry. Let $L_{1}, \ldots L_{n}$ be elements of $\mathcal{L}$ as in Theorem 1.8. For each $q \in Q$, let $\phi_{q}$ be a $(K, \epsilon)$-quasi-isometry such that $\partial \phi_{q}=q$. Let $\mathcal{F}_{0}=\left\{\phi_{q}: \partial \phi_{q} \in Q_{0}\right\}$.

By Theorem 1.8, there exists $C=C(K, \epsilon)$ such that $d\left(\phi_{q}(1), 1\right) \leq C$ for all $\phi_{q} \in \mathcal{F}_{0}$. Hence, by Lemma $1.4, \mathcal{F}_{0}$ is compact in the compact open topology on the space of self-maps of the dotted geodesic metric space consisting of the vertex set of $\Gamma$.

Suppose $\left\{\phi_{q_{i}}\right\} \subset \mathcal{F}_{0}$ is a sequence converging to $\phi_{q}$ in the compact open topology. Hence $q_{i}$ and $q$ are boundary values of $(K, \epsilon)$-quasi-isometries $\phi_{q_{i}}$ and $\phi_{q}$ respectively such that $\phi_{q_{i}}, \phi_{q}$ agree on larger and larger subsets as $i \rightarrow \infty$. It follows that $q_{i} \rightarrow q$ in Homeo( $\partial G$ ) (with the uniform topology) by stability of quasigeodesics (see Ghys and de la Harpe [27]) as $G$ is hyperbolic. 
Also, since $\operatorname{PP}(G, H)$ or $\operatorname{PPQI}(G, H)$ is a closed subgroup of $\operatorname{Homeo}(\partial G)$ equipped with the uniform topology, we have

Corollary 1.13 Let $H$ be an infinite quasiconvex subgroup of a hyperbolic group $G$ such that $H$ has infinite index. Let $\Gamma$ be a Cayley graph of $G$ with metric $d$ and $Q \subset \operatorname{PPQI}(G, H)$ be any group of boundary values of pattern-preserving uniform quasi-isometries of (the vertex set of) $\Gamma$. Then $Q$, equipped with the topology inherited from $\operatorname{Homeo}(\partial G)$, is locally compact.

Proof We include a slightly different direct proof here. The collection $\mathcal{L}$ is discrete by Proposition 1.10. Consider the finite collection $L_{1}, \ldots L_{n}$ in Corollary 1.12. There exists $\epsilon>0$ such that $N_{\epsilon}\left(L_{i}\right) \cap \mathcal{L}=L_{i}$ for all $i=1, \ldots, n$. Define

$$
N_{\epsilon}(\mathrm{Id})=\left\{q \in Q: d_{\partial G}(x, q(x)) \leq \epsilon \text { for all } x \in \partial G\right\},
$$

where $d_{\partial G}$ denotes some visual metric on $\partial G$. Then $N_{\epsilon}(\mathrm{Id}) \subset Q_{0}=\bigcap_{i=1, \ldots, n} \operatorname{Stab}\left(L_{i}\right)$, which is compact by Corollary 1.12 . Hence the Corollary.

\subsection{Boundaries of hyperbolic metric spaces and the Newman-Smith The- orem}

Let $L$ be one of the rings $\mathbb{Z}$ or $\mathbb{Z}_{p}$, for $p$ a prime.

Definition 1.14 [15, page 329] An m-dimensional homology manifold over $L$ (denoted $m-\mathrm{hm}_{L}$ ) is a locally compact Hausdorff space $X$ with finite homological dimension over $L$, that has the local homology properties of a manifold, ie for all $x \in X, H_{m}(X, X \backslash\{x\})=L$ and $H_{i}(X, X \backslash\{x\})=0$ for $i \neq m$.

Further, if $X$ is an $m-\mathrm{hm}_{L}$ and $H_{*}^{c}(X ; L) \cong H_{*}^{c}\left(\mathbb{S}^{m} ; L\right)$ then $X$ is called a generalized $m$-sphere over $L$. (Here $H_{*}^{c}$ denotes Čech homology and $H_{*}$ denotes ordinary singular homology.)

For homology manifolds, the existence of a local orientation was proven by Bredon [13].

The related notion of a cohomology manifold over $L$, denoted $m-\mathrm{cm}_{L}$ is defined by Bredon [15, page 373]. If $L=\mathbb{Z}_{p}$, a connected space $X$ is an $n-\mathrm{cm}_{L}$ if and only it if it is an $n-\mathrm{hm}_{L}$ and is locally connected [15, page 375, Theorem 16.8, footnote].

We shall be using the following Theorem which is a result that follows from work of Bestvina and Mess [4] and Bestvina [3] (see also Swenson [63], Bowditch [11] and Swarup [62]). 
Theorem 1.15 Boundaries $\partial G$ of $\operatorname{PD}(n)$ hyperbolic groups $G$ are locally connected homological manifolds (over the integers) with the homology of a sphere of dimension $(n-1)$. Further, if $G$ acts freely, properly discontinuously, cocompactly on a contractible complex $X$ then $H_{n}^{\mathrm{LF}}(X)=H_{n-1} \partial G$, where $H_{n}^{\mathrm{LF}}$ denotes locally finite homology.

In fact, one of the main results of [4] asserts that the (reduced) Čech cohomology groups of $\partial G$ vanish except in dimension $(n-1)$. Bestvina [3] also shows that the (reduced) Steenrod homology groups of $\partial G$ vanish except in dimension $(n-1)$. Since $\partial G$ is compact metrizable, Steenrod homology coincides with Čech homology (see for instance Milnor [43]). Further, for locally connected metrizable compacta such as $\partial G$, the Čech (co)homology groups coincide with singular (co)homology groups (see Lefschetz [40, page 107]). Hence the singular (as well as Čech) homology and cohomology of $\partial G$ coincide with that of a sphere of dimension $(n-1)$, ie $H_{0}(\partial G)=$ $H_{n-1}(\partial G)=H^{0}(\partial G)=H^{n-1}(\partial G)=\mathbb{Z}$ and all other homology and cohomology groups with $\mathbb{Z}$ coefficients are zero. Finally, using the Universal Coefficient Theorem for homology [34, Theorem 3A.3] $H_{i}\left(\partial G ; \mathbb{Z}_{p}\right)=H_{i}(\partial G) \otimes \mathbb{Z}_{p}$ since all the integral homology groups are torsion-free. Similarly for cohomology groups. Thus we have the following strengthening of Theorem 1.15:

Theorem 1.16 Let $L$ denote $\mathbb{Z}$, the integers or $\mathbb{Z}_{p}$, the integers $\bmod p$. Boundaries $\partial G$ of $\operatorname{PD}(n)$ hyperbolic groups $G$ are locally connected (co)homological manifolds (over $L$ ) with the (co)homology of a sphere of dimension $(n-1)$.

We shall be using Theorem 1.16 in conjunction with the following Theorem of Newman and Smith, which as stated below is a consequence of the work of several people (see below).

Theorem 1.17 (Newman [48], Smith [59]) Let $(X, d)$ be a compact $\mathbb{Z}_{p}$-cohomology manifold for all $p$, having finite topological (covering) dimension and equipped with a metric $d$. There exists $\epsilon>0$ such that (for any $n$ ) if $\mathbb{Z}_{n}$ acts effectively on $X$, then the diameter of some orbit is greater than $\epsilon$.

Newman proved the above Theorem for closed orientable manifolds [48]. Smith [59] generalized it to locally compact spaces satisfying certain homological regularity properties. Building on work of Yang [66], Conner and Floyd [21, Corollary 6.2] proved that cohomological manifolds of finite topological (covering) dimension satisfy the regularity properties required by Smith's theorem. (The theorem also holds for a somewhat more general class of spaces, called "finitistic spaces" by Bredon [14], but 
we shall not require this). For a historical account of the development of the theory of generalized/homology manifolds and their connection with Smith manifolds, see Raymond [52].

We shall also be using a theorem on homological consequences of actions of $p$-adic transformation groups on homology manifolds.

Theorem 1.18 (Yang [67]) Let $X$ be a compact homology $n$-manifold admitting an effective $K$-action, where $K=Z_{(p)}$ is the group of $p$-adic integers. Then the homological dimension of $X / K$ is $n+2$.

\section{Ahlfors regular metric measure spaces}

\subsection{General facts}

We refer to Heinonen [35] and Bonk and Kleiner [8] for details on quasisymmetric maps and metric measure spaces.

\section{Quasi-symmetric maps and quasi-isometries}

Definition 2.1 Let $f: X \rightarrow Y$ be a homeomorphism between metric spaces $\left(X, d_{X}\right)$ and $\left(Y, d_{Y}\right)$.

Then $f$ is $\eta$-quasisymmetric for some homeomorphism $\eta:[0, \infty) \rightarrow[0, \infty)$ if

$$
\frac{d_{Y}\left(f\left(x_{1}\right), f\left(x_{2}\right)\right)}{d_{Y}\left(f\left(x_{1}\right), f\left(x_{3}\right)\right)} \leq \eta\left(\frac{d_{X}\left(x_{1}, x_{2}\right)}{d_{X}\left(x_{1}, x_{3}\right)}\right)
$$

for every triple $\left(x_{1}, x_{2}, x_{3}\right)$ of distinct points in $X$.

The inverse of a quasisymmetric map is also quasisymmetric. In fact the right generalization of quasiconformal maps in $\mathbb{R}^{n}$ to metric measure spaces are quasisymmetric maps (cf [35, Chapters 10, 11]).

Proposition 2.2 [35, page 79] If $f: X \rightarrow Y$ is an $\eta$-quasisymmetric homeomorphism, then $f^{-1}: Y \rightarrow X$ is an $\eta_{1}$-quasisymmetric homeomorphism, where $\eta_{1}(t)=1 / \eta^{-1}\left(t^{-1}\right)$ for $t>0$. Further, if $f: X \rightarrow Y$ and $g: Y \rightarrow Z$ are $\eta_{f}$ and $\eta_{g}$-quasisymmetric homeomorphisms respectively, then $g \circ f: X \rightarrow Z$ is $\left(\eta_{g} \circ \eta_{f}\right)-$ quasisymmetric.

Remark 2.3 It follows from the definition of $\eta$-quasisymmetry that $d_{X}\left(x_{1}, x_{2}\right) \leq$ $d_{X}\left(x_{1}, x_{3}\right) \Rightarrow d_{Y}\left(f\left(x_{1}\right), f\left(x_{2}\right)\right) \leq \eta(1) d_{Y}\left(f\left(x_{1}\right), f\left(x_{3}\right)\right)$. Now let $B=B(x, r) \subset X$ denote the closed ball of radius $r$ about $x \in X$ and $\partial B=\left\{u \in B: d_{X}(x, u)=r\right\}$. Hence if $X$ is connected and $y, z \in \partial B$, then $d_{Y}(f(x), f(y)) \leq \eta(1) d_{Y}(f(x), f(z))$. 
Lemma 2.4 Let $g: X \rightarrow X$ be an $\eta$-quasisymmetric map of a compact metric space $(X, d)$ to itself. Then $g^{-1}$ is an $\eta_{1}$-quasisymmetric map by Proposition 2.2. Let $c=\eta_{1}^{-1}(1)$. Let $B=B(x, r)=\{y \in X: d(x, y) \leq r\}$ be the closed ball of radius $r$ about $x$. Let $\partial B=\{y \in X: d(x, y)=r\}$ denote the sphere of radius $r$ about $x$. Assume that $\partial B \neq \varnothing$. Let $s=d(g(x), g(\partial B))$. Then $B(g(x), c s) \subset g(B(x, r)$.

Proof It follows from compactness of $X$ that $\partial B$ is compact. Therefore $g(\partial B)$ is compact (and nonempty by hypothesis). Hence there exists $y_{0} \in \partial B$ such that $d\left(g(x), g\left(y_{0}\right)\right)=s$. Suppose $d(g(x), w) / d\left(g(x), g\left(y_{0}\right)\right) \leq c$ for some $w \in X$. Then $d\left(x, g^{-1}(w)\right) / d\left(x, y_{0}\right) \leq \eta_{1}(c)=1$. Hence $d\left(x, g^{-1}(w)\right) \leq d\left(x, y_{0}\right)=r$. Hence $w \in g(B)$.

It is a standard fact that the boundary values of quasi-isometries of proper hyperbolic metric spaces are quasisymmetric maps for any visual metric on the boundary:

Lemma 2.5 (Buyalo-Schroeder [18, Theorem 5.2.17, page 55], Väisälä [64, Theorem 5.35]) Suppose that $X$ and $Y$ are $\delta$-hyperbolic spaces equipped with basepoints and that $f: X \rightarrow Y$ is a base point preserving $(\lambda, \mu)$-quasi-isometry. Let $\left(\partial X, \rho_{X}\right)$ and $\left(\partial Y, \rho_{Y}\right)$ be their respective boundaries equipped with visual metrics. Then $f$ extends to an $\eta$-quasisymmetric homeomorphism $\partial f:\left(\partial X, \rho_{X}\right) \rightarrow\left(\partial Y, \rho_{Y}\right)$ with $\eta$ depending only on $\delta, \lambda, \mu$.

A converse result follows from work of Paulin [50]. We state it in the form we shall need it.

Theorem 2.6 Suppose that $X$ is (the Cayley graph with respect to a finite generating set of) a nonelementary hyperbolic group. Further suppose that $X$ is equipped with a basepoint. Let $Q$ be a compact group of $\eta$-quasisymmetric homeomorphisms in the uniform topology on $\partial X$. Then there exist $(\lambda, \mu)$ such that each $q \in Q$ may be realized as the boundary value of a $(\lambda, \mu)$-quasi-isometry of $X$ fixing the basepoint.

Proof By Paulin's work [50], there exist $\lambda_{1}, \mu_{1}$ such that every element $q \in Q$ may be realized as the boundary value of a $\left(\lambda_{1}, \mu_{1}\right)$-quasi-isometry of $X$. We denote the map induced by $q$ on $\left(\partial^{3} X, \rho\right)$ by $q^{3}$. Here $\left(\partial^{3} X, \rho\right)$ is the pseudo-metric space in Observation 1.5.

Since $X$ is quasi-isometric to $\left(\partial^{3} X, \rho\right)$ for the dotted path-metric constructed on the set of distinct triples (Observation 1.5), every element $q \in Q$ may be realized as the boundary value of a uniform quasi-isometry $q^{3}$ of $\left(\partial^{3} X, \rho\right)$. 
Since $Q$ is compact, any $q^{3} \in Q$ maps a fixed triple $\left(a_{1}, a_{2}, a_{3}\right) \in\left(\partial^{3} X, \rho\right)$ to triples that are uniformly separated in $(\partial X, d)$ where $d$ denotes a visual metric, ie there exists $\epsilon>0$ such that for all $q \in Q, d\left(q\left(a_{i}\right), q\left(a_{j}\right)\right)>\epsilon$ for $i \neq j$. Hence $\rho\left(\left(a_{1}, a_{2}, a_{3}\right), q^{3}\left(a_{1}, a_{2}, a_{3}\right)\right)$ is uniformly bounded. Choose $\left(a_{1}, a_{2}, a_{3}\right) \in\left(\partial^{3} X, \rho\right)$ as the basepoint in $\left(\partial^{3} X, \rho\right)$.

Define a map $\phi_{q}:\left(\partial^{3} X, \rho\right) \rightarrow\left(\partial^{3} X, \rho\right)$ as follows:

(1) $\phi_{q}$ maps $q^{3}\left(a_{1}, a_{2}, a_{3}\right)$ and all points at $\rho$ distance zero from it to $\left(a_{1}, a_{2}, a_{3}\right)$.

(2) $\phi_{q}$ fixes all other points of $\left(\partial^{3} X, \rho\right)$.

Then $\phi_{q} \circ q^{3}$ is a uniform (independent of $\left.q\right)$ quasi-isometry of $\left(\partial^{3} X, \rho\right)$ fixing a basepoint. postcomposing further by the inverse of the quasi-isometry from $X$ to $\left(\partial^{3} X, \rho\right)$ and moving back a basepoint in $X$ again if necessary, we get the required result.

\section{Ahlfors regular and doubling spaces}

Definition 2.7 A metric space $X$ is said to be doubling if for all $\lambda \geq 1$ there exists $N \in \mathbb{N}$ such that for all $x \in X$ the ball $\lambda B(x, r)=B(x, \lambda r)$ can be covered by $N$ balls of radius $r$.

We shall need a special case of a Theorem of Bonk and Schramm [9].

Theorem 2.8 (Bonk-Schramm [9]) Let $X$ be a Gromov-hyperbolic group or a complete simply connected Hadamard manifold of pinched negative curvature. Then $(\partial X, d)$ is doubling for any visual metric $d$.

Definition 2.9 Let $(X, d)$ be a metric space. Then the $k$-dimensional Hausdorff measure of $X$ is defined by

$$
\inf \left\{\sum_{i} r_{i}^{k} \text { : there exists a cover of } X \text { by balls } B_{i} \text { of radius } r_{i}\right\} \text {. }
$$

The Hausdorff dimension $\operatorname{dim}_{\text {haus }}(X)$ of $X$ is defined to be the infimum of all $k \geq 0$ such that the $k$-dimensional Hausdorff measure of $X$ is zero.

In this paper we shall have occasion to use the easier to compute notion of Minkowski dimension where the balls used to cover $X$ are of equal radii. See Sullivan [60], Coornaert [22] and Bishop and Jones [5] for the equivalence in our situation (also see Falconer [25] for very general sufficient conditions ensuring equality of Hausdorff and Minkowski dimensions). 
Definition 2.10 Let $(X, d, \mu)$ be a compact metric measure space, ie a metric space equipped with a Borel measure. We say that $X$ is Ahlfors $Q$-regular, if there exists $C_{0} \geq 1$ such that for all $0 \leq r \leq \operatorname{diam}(X)$, and any ball $B_{r}(x) \subset X$, the measure $\mu\left(B_{r}(x)\right)$ satisfies $\left(1 / C_{0}\right) r Q \leq \mu\left(B_{r}(x)\right) \leq C_{0} r Q$.

When $Q$ is omitted in Definition 2.10, we assume that $Q$ is the Hausdorff dimension and $\mu$ the Hausdorff measure.

The relevance to the present paper comes from the following.

Theorem 2.11 (Coornaert [22]) Let $G$ be a hyperbolic group. Then $(\partial G, d, \mu)$ is Ahlfors regular for any visual metric $d$ and the associated Hausdorff measure $\mu$.

Certain very general conditions ensure Ahlfors regularity. Corollary 14.15 of [35] asserts that a metric space is quasisymmetrically equivalent to an Ahlfors regular space if and only if it is uniformly perfect and carries a doubling measure. We shall not be needing the precise definitions of these terms. Suffice to say that a metric space carries a doubling measure if and only if it is doubling [41;20]; and connected sets are uniformly perfect. However for our purposes, the proof of the more restrictive Theorem 5.4 of [22] for hyperbolic groups which in turn is modelled on Sullivan's work [60] suffices to ensure the following. (See [60, Theorem 25] in particular which devotes special attention to noncompact finite volume manifolds or [51, Theorem 0.2].)

Theorem 2.12 Let $X$ be the universal cover of a complete finite volume manifold of pinched negative curvature. Then $(\partial X, d, \mu)$ is Ahlfors regular for any visual metric $d$ and the associated Hausdorff measure $\mu$.

The following notion is essentially due to Bourdon and Pajot [10].

Definition 2.13 Let $\Gamma$ be a hyperbolic group or more generally a hyperbolic metric space. We define the Ahlfors regular conformal dimension of the boundary of $\Gamma$ as

$\operatorname{ACD}(\partial \Gamma)=\inf \{Q: \partial \Gamma$ admits a visual metric with Hausdorff dimension $Q\}$.

In this paper we shall repeatedly make the assumption that the boundary $\partial \Gamma$ of $\Gamma$ admits a visual metric $d$ such that the Hausdorff dimension $\operatorname{dim}_{\text {haus }}$ of $(\partial \Gamma, d)$ is less than $\left(\operatorname{dim}_{t}+2\right)$, where $\operatorname{dim}_{t}$ denotes topological dimension. Note that by Definition 2.13 above, this is equivalent to saying that $\operatorname{ACD}(\partial \Gamma)<\left(\operatorname{dim}_{t}(\partial \Gamma)+2\right)$. 
Also note that all visual metrics are quasisymmetrically conjugate to each other. The identity map on the underlying topological space gives the required quasisymmetry. More generally, for a metric space $X, \operatorname{ACD}(X)$ is usually defined [10] by

$\operatorname{ACD}(X)=\inf \{Q:$ there exists an Ahlfors $Q$-regular metric space $Y$

that is quasisymmetrically equivalent to $X$ \}.

We shall not have need for this more general notion in this paper.

We collect certain notions and facts from Bonk and Kleiner [8] and Heinonen [35] that we shall have need for in what follows.

Lemma 2.14 [35, Theorem 1.2, page 2] Every family $\mathcal{F}$ of balls of uniformly bounded diameter in a metric space $X$ contains a pairwise disjoint subfamily $\mathcal{G}$ such that $\bigcup_{B \in \mathcal{F}} B \subset \bigcup_{B \in \mathcal{G}} 5 B$, where $\lambda B$ denotes a ball concentric with $B$ and radius $\lambda$ times that of $B$.

Corollary 2.15 Let $X$ be a doubling metric space. There exists an $M$ such that the following holds.

Let $\mathcal{F}_{r}$ be the family of all balls of radius $r$ in $X$. Let $\mathcal{G}_{r}$ be any pairwise disjoint subfamily such that $X=\bigcup_{B \in \mathcal{G}_{r}} 5 B$. Then for any $x \in X$, the cardinality of the set $\left\{B \in \mathcal{G}_{r}: x \in 5 B\right\}$ is less than or equal to $M$.

Proof We omit the suffix $r$ for convenience. The existence of a pairwise disjoint subfamily $\mathcal{G}$ of $\mathcal{F}$ such that $X=\bigcup_{B \in \mathcal{G}} 5 B$ is guaranteed by Lemma 2.14.

We now argue by contradiction. Suppose that no such $M$ exists. Then for all $N \in \mathbb{N}$, there exists $r_{N}>0$, a pairwise disjoint subfamily $\mathcal{G}$ of balls of radius $r_{N}$ in $X$, and an $x \in X$ such that the cardinality of the set $\{B \in \mathcal{G}: x \in 5 B\}$ is greater than $N$. Hence the $6 r_{N}$ ball around $x$ contains more than $N$ distinct (and hence disjoint) elements of $\mathcal{G}$. Since $N$ is arbitrary, this contradicts the hypothesis that $X$ is doubling.

We start with a general result about compact group actions on doubling, Ahlfors regular compact metric measure spaces, eg boundaries of one-ended hyperbolic groups.

Lemma 2.16 Let $(X, d, \mu)$ be a connected, doubling, Ahlfors regular compact metric measure space having Hausdorff dimension $\mathcal{Q} \geq 1$ (for instance the boundary of a one-ended hyperbolic group or the boundary of the universal cover of a finite volume manifold of pinched negative curvature by Theorems 2.82 .11 and 2.12). Let $K$ be a compact topological group acting by uniformly $\eta$-quasisymmetric maps on $(X, d, \mu)$ and equipped with a Haar measure of unit mass. Let $C=\eta(1), c=\eta_{1}^{-1}(1)$, and let $d_{K}$ be the average metric on $X$ given by $d_{K}(x, y)=\int_{K} d(g(x), g(y)) d g$. Then the Hausdorff dimension of $\left(X, d_{K}, \mu\right)$ does not exceed $\mathcal{Q}$. 


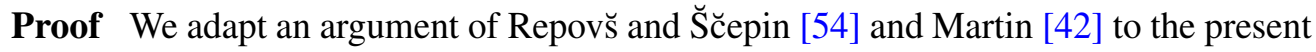
context. Assume after normalization, that the total Hausdorff measure of $X$ is one.

Cover $X$ by a family of balls of radius $r>0$ measured with respect to $d$. By Lemma 2.14 we can choose a family $\mathcal{B}_{r}$ of pairwise disjoint balls of radius $r$ such that $X=\bigcup_{B \in \mathcal{B}_{r}} 5 B$. As in [42], it is enough to find a uniform bound (independent of $r$ ) for the sum $\sum_{B \in \mathcal{B}_{r}}\left(\operatorname{diam}_{d_{K}} 5 B\right)^{\mathcal{Q}}$ where $\operatorname{diam}_{d_{K}} 5 B$ is the diameter of $5 B$ in the invariant metric $d_{K}$.

For each $B \in \mathcal{B}_{r}$ let $c_{B}$ denote its center and let $z_{B}$ be a point on the boundary of $5 B$ with $\operatorname{diam}_{d_{K}} B \leq 2 d_{K}\left(c_{B}, z_{B}\right)$. (Such a point $z_{B}$ exists as $X$ is connected.) Then

$$
\begin{aligned}
\sum_{B \in \mathcal{B}_{r}}\left(\operatorname{diam}_{d_{K}} 5 B\right)^{\mathcal{Q}} & \leq 2^{\mathcal{Q}} \sum_{B \in \mathcal{B}_{r}}\left(\int_{K} d\left(g\left(c_{B}\right), g\left(z_{B}\right)\right) d g\right)^{\mathcal{Q}} \\
& \leq 2^{\mathcal{Q}} \sum_{B \in \mathcal{B}_{r}} \int_{K} d\left(g\left(c_{B}\right), g\left(z_{B}\right)\right)^{\mathcal{Q}} d g \\
& \leq(2 C)^{\mathcal{Q}} \sum_{B \in \mathcal{B}_{r}} \int_{K} \inf _{y_{B} \in \partial(5 B)} d\left(g\left(c_{B}\right), g\left(y_{B}\right)\right)^{\mathcal{Q}} d g \\
& =\left(\frac{2 C}{c}\right)^{\mathcal{Q}} \sum_{B \in \mathcal{B}_{r}} \int_{K}\left(c \inf _{y_{B} \in \partial B} d\left(g\left(c_{B}\right), g\left(y_{B}\right)\right)\right)^{\mathcal{Q}} d g
\end{aligned}
$$

where the second line holds by Holder's inequality since $\mathcal{Q} \geq 1$ and the normalization condition that the total Haar measure of $K$ is one, the third lines holds since $K$ acts by uniformly $\eta$-quasisymmetric maps, and since $\partial(5 B) \neq \varnothing$ by connectedness of $X$ and the last equality holds by Remark 2.3.

But by Lemma 2.4, the ball of radius $c \inf _{y_{B} \in \partial B} d\left(g\left(c_{B}\right), g\left(y_{B}\right)\right)$ is contained in $g(5 B)$. Hence

$$
\begin{aligned}
\left(\frac{2 C}{c}\right)^{\mathcal{Q}} \sum_{B \in \mathcal{B}_{r}} \int_{K}\left(c \inf _{y_{B} \in \partial B} d\left(g\left(c_{B}\right), g\left(y_{B}\right)\right)\right)^{\mathcal{Q}} d g & \leq C_{0}\left(\frac{2 C}{c}\right)^{\mathcal{Q}} \sum_{B \in \mathcal{B}_{r}} \int_{K} \mu(g(5 B)) d g \\
& =C_{0}\left(\frac{2 C}{c}\right)^{\mathcal{Q}} \int_{K} \sum_{B \in \mathcal{B}_{r}} \mu(g(5 B)) d g \\
& \leq C_{0} M\left(\frac{2 C}{c}\right)^{\mathcal{Q}} \int_{K} \mu(g(X)) d g,
\end{aligned}
$$

where $C_{0}$ is the constant appearing in the definition of Ahlfors $\mathcal{Q}$-regularity of $X$ and $M$ is the constant appearing in Corollary 2.15 giving an upper bound for the local multiplicity of the cover $\left\{5 B: B \in \mathcal{B}_{r}\right\}$. 
Since $g$ is a homeomorphism from $X$ onto itself, this equals

$$
C_{0} M\left(\frac{2 C}{c}\right)^{\mathcal{Q}} \int_{K} \mu(X) d g=C_{0} M\left(\frac{2 C}{c}\right)^{\mathcal{Q}},
$$

where the equality holds since $K$ is equipped with a Haar measure of unit mass and by the normalization condition that the total Hausdorff measure of $X$ is one.

This establishes a uniform bound (independent of $r$ ) for the sum $\sum_{B \in \mathcal{B}_{r}}\left(\operatorname{diam}_{d_{K}} 5 B\right)^{\mathcal{Q}}$ and completes the proof.

Theorem 2.17 (See [36, Chapter II, Theorems 7.1-7.6, pages 60-61].) Let $G$ be a topological group. Then there exists an exact sequence

$$
1 \rightarrow G_{0} \rightarrow G \rightarrow H \rightarrow 1
$$

with $G_{0}$ the connected component of the identity in $G$ and $H$ totally disconnected. If $G$, and hence $H$, is locally compact, then $H$ contains arbitrarily small compact open subgroups (ie for every neighborhood $U$ of the identity in $H$, there is a compact open subgroup $K$ contained in $U$ ).

Moreover the structure of $G_{0}$ is well-known thanks to Montgomery and Zippin [45, Theorem 4.6] and Gleason [29].

We state a definition first.

Definition 2.18 A topological group is said to have arbitrarily small torsion elements if for every neighborhood $U$ of the identity, there exists an element $g \in U, g \neq 1$, and a positive integer $n$ such that $g^{n}=1$, and furthermore $g^{m} \in U$ for all $m \in \mathbb{N}$.

A topological group is said to have small subgroups if for every neighborhood $U$ of the identity, there exists a subgroup $H \neq\{1\}$ such that $H$ is contained in $U$.

The following is a celebrated Theorem of Montgomery and Zippin [45] and Gleason [29].

Theorem 2.19 [45, Sections 4.5, 4.6, 4.9; 29] Let $G_{0}$ be the connected component of the identity in a locally compact topological group $G$ such that $G / G_{0}$ is compact. For each neighborhood $U$ of the identity in $G_{0}$, there exists a compact normal subgroup $K \subset U$ such that the quotient group $G_{0} / K$ is a Lie group.

Small subgroups of connected locally compact finite dimensional groups are totally disconnected and belong to the center.

Any locally compact finite dimensional group with no small subgroups is a Lie group. 
Remark 2.20 The hypothesis in Theorem 2.19 that $G / G_{0}$ is compact is superfluous. Let $H=G / G_{0}$ and $q: G \rightarrow H$ be the quotient homomorphism. By Theorem 2.17, $H$ contains a compact open totally disconnected subgroup $H_{1}$. Let $G_{1}=q^{-1}\left(H_{1}\right)$. Then $G_{0}$ is the connected component of the identity of $G_{1}$ and $G_{1} / G_{0}$ is compact. Theorem 2.19 now applies.

We also have the following Theorem of Yamabe [65].

Theorem 2.21 (Yamabe [65]) A group with a neighborhood $U$ of the identity which does not contain any nontrivial normal subgroup has a neighborhood $V$ of the identity which does not contain any nontrivial subgroup.

Definition 2.22 A topological group containing a cyclic dense subgroup is said to be monothetic.

It is easy to see that a monothetic group is abelian (using continuity of multiplication). The following is a consequence of a structure theorem for 0-dimensional compact monothetic groups (see [36, Theorem 25.16, page 408]).

Theorem 2.23 Any infinite 0-dimensional (ie totally disconnected), compact, monothetic group $K$ contains a copy of the a-adic integers $A_{\mathbf{a}}$, where $\mathbf{a}=\left\{a_{1}, a_{2}, \ldots\right\}$ is a sequence of integers $a_{i}>1$. Hence $K$ must contain arbitrarily small torsion elements or a copy of the group $Z_{(p)}$ of $p$-adic integers.

\subsection{The Hilbert-Smith Conjecture}

Theorem 2.24 Let $(X, d, \mu)$ be a connected, doubling, Ahlfors regular compact metric measure space. Further suppose that $X$ is a $\mathbb{Z}_{p}$-cohomology manifold for all $p$; and $1 \leq \operatorname{dim}_{\text {haus }}(X)<\operatorname{dim}_{h}(X)+2$, where $\operatorname{dim}_{\text {haus }}$ is the Hausdorff dimension and $\operatorname{dim}_{h}$ is the homological dimension. Then $(X, d, \mu)$ does not admit an effective $Z_{(p)}$-action by uniform quasisymmetric maps, where $Z_{(p)}$ denotes the $p$-adic integers. Hence any finite dimensional locally compact group acting effectively on $(X, d, \mu)$ by uniform quasisymmetric maps is a Lie group.

Proof Let $K=Z_{(p)}$ be the compact group of $p$-adic integers acting effectively on $X$ by uniform quasisymmetric maps. Let $d_{K}$ be the average metric on $X$ given by $d_{K}(x, y)=\int_{K} d(g(x), g(y)) d g$. By Lemma 2.16 the Hausdorff dimension of $\left(X, d_{K}, \mu\right)$ does not exceed $\operatorname{dim}_{\text {haus }}(X)$. Then $K$ acts on $\left(X, d_{K}\right)$ by isometries. Therefore, the orbit space $X / K$ admits the well-defined metric $\rho([x],[y])=$ $d_{K}(K(x), K(y))$, where $[x],[y]$ denote the images of $x, y$ under the quotient map 
by $K$. Let $P: X \rightarrow X / K$ be the natural quotient map. Since $P$ is clearly $1-$ Lipschitz, it cannot increase Hausdorff dimension. Hence the Hausdorff dimension of $X / K$ is at most equal to $\operatorname{dim}_{\text {haus }}(X)$, the Hausdorff dimension of $X$, which in turn is less than $\operatorname{dim}_{h}(X)+2$. Since topological dimension is majorized by Hausdorff dimension and homological dimension is majorized by topological dimension, it follows that the homological dimension of $X / K$ is less than $\operatorname{dim}_{h}(X)+2$. This directly contradicts Yang's Theorem 1.18 which asserts that the homological dimension of $X / K$ is equal to $\operatorname{dim}_{h}(X)+2$ and establishes the first part of the theorem.

The last statement follows from the first by standard arguments (see [54; 42], for instance). We outline the argument for the sake of completeness.

Let $G$ be any finite dimensional locally compact group acting effectively on $X$ by uniform quasisymmetric maps. By the last statement of Theorem 2.19, if $G$ has no small subgroups, then $G$ must be a Lie group.

We now proceed by contradiction. If possible, let $G$ have small subgroups. By local compactness, we may assume that $G$ has compact small subgroups. Hence by Yamabe's Theorem 2.21, $G$ has a sequence $\left(K_{i}\right)_{i}$ of compact normal small subgroups such that $K_{i} \neq\{1\}$ for all $i$ and such that $\bigcap_{i} K_{i}=\{1\}$. Let $L$ be the connected component of the identity of $G$. Then either $K_{i} \cap L \neq\{1\}$ or $K_{i}$ is totally disconnected. If $K_{i} \cap L \neq\{1\}$ then $K_{i} \cap L$ is a compact normal small subgroup of $L$. Hence again by the second statement of Theorem $2.19, K_{i} \cap L$ is totally disconnected. In any case, $G$ has a sequence $\left(K_{i}\right)_{i}$ of nontrivial compact normal totally disconnected small subgroups.

If $K$ is infinite, it must contain a copy of the $p$-adics (Theorem 2.23) or have arbitrarily small torsion elements. By Theorem $1.17, K$ cannot have arbitrarily small torsion elements. Hence $Z_{(p)}$ acts effectively on $X$ by uniform quasisymmetric maps, contradicting the first assertion of the Theorem proved above.

Since quasi-isometries of a hyperbolic group $G$ act by quasisymmetric maps on the boundary $(\partial G, d)$, where $d$ is a visual metric, we have the following by combining Theorems 1.16 and 2.11 with Theorem 2.24.

Corollary 2.25 Let $\Gamma$ be a Poincare duality hyperbolic group and $Q$ be a group of (boundary values of) quasi-isometries of $\Gamma . Q$ is equipped with the uniform topology. Suppose $d$ is a visual metric on $\partial \Gamma$ with $\operatorname{dim}_{\text {haus }}<\operatorname{dim}_{t}+2$, where $\operatorname{dim}_{\text {haus }}$ is the Hausdorff dimension and $\operatorname{dim}_{t}$ is the topological dimension of $X=(\partial \Gamma, d)$. Equivalently suppose that $\operatorname{ACD}(X)<\operatorname{dim}_{t}+2$ where $\operatorname{ACD}(X)$ denotes the Ahlfors regular conformal dimension of $X$. Then $Q$ cannot contain a copy of $Z_{(p)}$, where $Z_{(p)}$ denotes the $p$-adic integers. Hence if $Q$ is finite dimensional locally compact, it must be a Lie group. 
Proof By Theorem 1.16, $X$ is a $\mathbb{Z}_{p}$-cohomology manifold for all $p$. By Theorems 2.8 and 2.11 and by one-endedness of $\Gamma, X=(\partial \Gamma, d, \mu)$ is a connected, doubling, Ahlfors regular compact metric measure space.

The assumption of Theorem 2.24 that $1 \leq \operatorname{dim}_{\text {haus }}$ is superfluous here as the only PD group that violates this hypothesis is virtually cyclic, when the Corollary is trivially true.

If possible, let $K=Z_{(p)}$ be the compact group of $p$-adic integers acting effectively on $X$ by quasisymmetric maps. All that remains to be shown is that we can extract an action of $Z_{(p)}$ by uniform quasisymmetric maps.

Let $\left(\partial^{3} X, \rho\right)$ be the pseudo-metric space in Observation 1.5. Fix a basepoint $x \in \partial^{3} X$. Since $K$ is compact, $\rho\left(k^{3}(x), x\right)$ is uniformly bounded.

For each $k \in K$ there exist $\lambda_{k} \in \mathbb{N}$ such that $k^{3}:\left(\partial^{3} X, \rho\right) \rightarrow\left(\partial^{3} X, \rho\right)$ is a $\left(\lambda_{k}, \lambda_{k}\right)-$ quasi-isometry. Note further that $q_{n} \rightarrow q$ in the uniform topology implies that $q_{n}^{3} \rightarrow q^{3}$ in the compact open topology.

Let $U_{i}=\left\{k \in K, \lambda_{k} \leq i\right\}$. Then $K=\bigcup_{i} \overline{U_{i}}$ is the union of a countable family of closed sets $\overline{U_{i}}$. Note further that the limit of a sequence of $\left(\lambda_{k}, \lambda_{k}\right)$-quasi-isometries is (at most) a $\left(\lambda_{k}+2, \lambda_{k}+2\right)$ quasi-isometry. By the Baire category theorem there is some $U_{C}$ with nonempty interior. Translating by an element $h$ of $K$ in the interior of $U_{C}$, we may assume that $U_{c}$ (for some $c$ depending on $C$ and the quasi-isometry constant of $h$ ) contains the identity. But any neighborhood of the identity in $K$ contains an isomorphic copy of $K$.

Hence we have an action of $K$ (replacing the original group by the isomorphic copy contained in the above neighborhood of the identity) on $\left(\partial^{3} X, \rho\right)$ by $(c, c)$-quasiisometries. Since $(X=\partial \Gamma, d)$ is a visual boundary for $\left(\partial^{3} X, \rho\right)$ and since each element in $K$ moves the basepoint $x$ by a uniformly amount, we have an action of $K$ on $(X, d, \mu)$ by uniformly quasisymmetric maps by Lemma 2.5. Theorem 2.24 now furnishes the required conclusion.

Note The hypothesis $\operatorname{dim}_{\text {haus }}<\operatorname{dim}_{t}+2$ (or $\operatorname{ACD}(X)<\operatorname{dim}_{t}+2$ ) is clearly true for (uniform lattices in) real hyperbolic space, where $\operatorname{dim}_{\text {haus }}=\operatorname{dim}_{t}$ as well as complex hyperbolic space, where $\operatorname{dim}_{\text {haus }}=\operatorname{dim}_{t}+1$. Amongst rank one symmetric spaces these are the only ones of interest in the context of pattern rigidity as quaternionic hyperbolic space and the Cayley plane are quasi-isometrically rigid in light of Pansu's fundamental result [49]. 


\section{Pattern preserving groups as topological groups}

\subsection{Infinite divisibility}

We begin with some easy classical facts about topological groups. A property we shall be investigating in some detail is the notion of "topological infinite divisibility". The notion we introduce is weaker than related existing notions in the literature.

Definition 3.1 An element $g$ in a topological group $G$ will be called topologically infinitely divisible, if there exists a sequence of symmetric neighborhoods $\left(U_{k}\right)_{k}$ of the identity, such that $\bigcap_{k} U_{k}=\{1\}$ and $g \in \bigcup_{n=1}^{\infty} U_{k}^{n} \subset G$ for all $k$. Similarly, a subgroup $H$ of $G$ is said to be topologically infinitely divisible, if there exists a sequence of symmetric neighborhoods $\left(U_{k}\right)_{k}$ of the identity, such that $\bigcap_{k} U_{k}=\{1\}$ and $H \subset \bigcup_{1}^{\infty} U_{k}^{n} \subset G$ for all $k$.

Let $\Gamma$ be a hyperbolic group and $H$ an infinite quasiconvex subgroup of infinite index in $\Gamma$. Then the collection of translates of the (join of the) limit set of $H$ in $\partial \Gamma$ gives rise to a symmetric pattern.

For the rest of this subsection $Q$ will denote a group of boundary values of patternpreserving quasi-isometries of (the Cayley graph of) $G$.

Recall that the topology on $Q$ is inherited from the uniform topology on Homeo( $\partial \Gamma)$. Also recall that $q \in Q$ is a quasisymmetric map on the boundary $\partial \Gamma$.

Lemma 3.2 $q_{n} \rightarrow q$ in the uniform topology on Homeo( $\left.\partial \Gamma\right)$ if and only if $q_{n}^{3} \rightarrow q^{3}$ in the compact-open topology on $\partial^{3} \Gamma$.

Proof Suppose $q_{n} \rightarrow q$ in the uniform topology on Homeo( $(\partial \Gamma)$. Then $q_{n}^{3} \rightarrow q^{3}$ in the uniform topology on $\operatorname{Homeo}(\partial \Gamma \times \partial \Gamma \times \partial \Gamma)$. Since $\partial^{3} \Gamma$ is an open invariant subset of $\partial \Gamma \times \partial \Gamma \times \partial \Gamma$, it follows that $q_{n}^{3} \rightarrow q^{3}$ on compact subsets of $\partial^{3} \Gamma$. Hence $q_{n}^{3} \rightarrow q^{3}$ in the compact-open topology on $\partial^{3} \Gamma$.

Next suppose $q_{n}^{3} \rightarrow q^{3}$ in the compact-open topology on $\partial^{3} \Gamma$. If $q_{n}$ does not converge to $q$ uniformly on $\partial \Gamma$, then (after passing to a subsequence if necessary) there exists $\epsilon>0$ such that for all $n$ there exists $x_{n} \in \partial \Gamma$ such that $d\left(q_{n}\left(x_{n}\right), q\left(x_{n}\right)\right) \geq 2 \epsilon$. Passing to a further subsequence if necessary we can assume that $x_{n} \rightarrow x \in \partial \Gamma$. Hence $q\left(x_{n}\right) \rightarrow q(x) \in \partial \Gamma$. Hence by passing to a further subsequence if necessary we can assume that $d\left(q_{n}\left(x_{n}\right), q(x)\right) \geq \epsilon$ for all $n$. Choosing $y, z$ fixed distinct points unequal to $x$, it follows that $q_{n}^{3}\left(x_{n}, y, z\right)$ does not converge to $q^{3}(x, y, z)$, a contradiction. 
Proposition 3.3 $Q$ has no nontrivial topologically infinitely divisible elements. More generally, $Q$ does not contain any nontrivial infinitely divisible subgroups.

Proof Suppose $q \in Q$ is infinitely divisible. Then for every neighborhood $U$ of 1 there exists $m \in \mathbb{N}$ such that $q \in U^{m}$. Now for any finite collection $L_{1}, \ldots, L_{n} \in \mathcal{L}$, there exists a neighborhood $U$ of $1 \in Q$ such that if $g \in U$ then $g\left(L_{i}\right)=L_{i}$ for $i=1, \ldots, n$.

Hence for any finite collection $L_{1}, \ldots, L_{n} \in \mathcal{L}$, there exists a neighborhood $U$ of 1 and $m \in \mathbb{N}$ such that

(1) $q \in U^{m}$,

(2) $g\left(L_{j}\right)=L_{j}$ for $g \in U, j=1, \ldots, n$.

From (2) it follows that $g\left(L_{j}\right)=L_{j}$ for $g \in U^{m}, j=1, \ldots, n$ and all $m \in \mathbf{N}$.

Therefore $q\left(L_{j}\right)=L_{j}$ for all $L_{j} \in \mathcal{L}$. That is $q$ stabilizes every $L \in \mathcal{L}$. If $x \in \partial \Gamma$, there exist $L_{m} \in \mathcal{L}$ such that $L_{m} \rightarrow\{x\}$ (the singleton set containing $x$ ) in the Hausdorff topology on $\partial \Gamma$. Therefore $q(\{x\})=\{x\}$ for all $x \in \partial \Gamma$, ie $q$ is the trivial element of $Q$. The same argument shows that $Q$ has no nontrivial topologically infinitely divisible subgroups.

Remark 3.4 We state the second conclusion of Proposition 3.3 slightly differently. Let $U_{i}$ be a decreasing sequence of symmetric neighborhoods of the identity in $Q$ such that $\bigcap_{i} U_{i}=\{1\}$. Let $\left\langle U_{i}\right\rangle=\bigcup_{n} U_{i}^{n}$. Then $\bigcap_{i}\left\langle U_{i}\right\rangle=\{1\}$.

Since a connected topological group is generated by any neighborhood of the identity, we obtain:

Proposition 3.5 $Q$ is totally disconnected.

Note that in Proposition 3.3 and Proposition 3.5 we do not need to assume that $Q$ is a group of uniform quasi-isometries.

Remark 3.6 Let $K$ be a compact group of pattern-preserving quasi-isometries. Then a reasonably explicit structure of $K$ may be given as a permutation group. Since $K$ is compact it acts on the discrete set $\mathcal{L}$ with compact and hence finite orbits. Let $\mathcal{L}_{1}, \mathcal{L}_{2}, \ldots$ be a decomposition of $\mathcal{L}$ into disjoint orbits under $K$. Then $K \subset \Pi_{i} S\left(\mathcal{L}_{i}\right)$, where $S\left(\mathcal{L}_{i}\right)$ denotes the symmetric group on the finite set $\mathcal{L}_{i}$ and $\Pi$ denotes direct product. Thus, we have a natural representation of $K$ as a permutation group on an infinite set, where every orbit is finite. The last part of the argument in Proposition 3.3 shows that this representation is faithful, since any element stabilizing every element of $\mathcal{L}$ must be the identity. 


\subsection{PD groups}

Boundaries $\partial G$ of $\operatorname{PD}(n)$ hyperbolic groups $G$ are locally connected homological manifolds (over the integers) with the homology of a sphere of dimension $(n-1)$ by Theorem 1.16. The interested reader may refer to Davis' survey [23] for background on PD groups.

If $\mathrm{QI}(G)$ denotes the group of boundary values of quasi-isometries of $G$ acting on $\partial G$ (equipped with the uniform topology), then Theorem 1.17 implies the following.

Proposition 3.7 $\mathrm{QI}(G)$ cannot have arbitrarily small torsion elements.

We combine Corollary 2.25 with Proposition 3.7 to get the following.

Proposition 3.8 Let $G$ be a Poincare duality hyperbolic group and $H$ an infinite quasiconvex subgroup of infinite index in $G$. Let $K$ be a compact group of (boundary values of) pattern-preserving quasi-isometries of $G$. Suppose $d$ is a visual metric on $\partial G$ with $\operatorname{dim}_{\text {haus }}<\operatorname{dim}_{t}+2$, where $\operatorname{dim}_{\text {haus }}$ is the Hausdorff dimension and $\operatorname{dim}_{t}$ is the topological dimension of $(\partial G, d)$. Equivalently suppose that $\operatorname{ACD}(\partial G)<\operatorname{dim}_{t}+2$ where $\operatorname{ACD}(\partial G)$ denotes the Ahlfors regular conformal dimension of $\partial G$. Then $K$ must be finite.

Proof By Proposition 3.7, $K$ cannot have arbitrarily small torsion elements. Hence there exists $\epsilon>0$ such that for any nontrivial $k \in K$, there exists an $x \in \partial G$ such that $\langle k\rangle x$ has diameter greater than $\epsilon$ where $\langle k\rangle$ denotes the cyclic group generated by $k$. Again, since $K$ is infinite and compact there exists a sequence of distinct elements $k_{i} \rightarrow 1$ in $K$. By compactness of $\partial G$, there exists $x \in \partial G$, such that $\left\langle k_{i}\right\rangle x$ has diameter greater than $\epsilon$ and hence the order $o\left(k_{i}\right) \rightarrow \infty$ as $i \rightarrow \infty$.

Therefore the subgroups $\left\langle k_{i}\right\rangle \subset K$ converge up to a subsequence (in the Chabauty topology on closed subgroups of $K$ ) to an infinite compact nontrivial abelian group $L$ without small torsion elements. If $L$ is pure torsion it must have elements of arbitrarily large order (since $L$ being infinite and compact must contain elements arbitrarily close to the identity). This contradicts the structure of compact abelian torsion groups (see [36, Theorem 25.9]).

Hence, if $K$ is infinite, it must have an element $g$ of infinite order. Let $C(g)$ be the (closed) monothetic subgroup generated by $g$. Since $K$ is totally disconnected by Proposition 3.5 so is $C(g)$ and hence $C(g)$ cannot have arbitrarily small torsion elements. By Theorem $2.23 C(g)$ must contain a copy of the $p$-adic integers. But $K$ cannot contain a copy of the $p$-adic integers by Corollary 2.25, a contradiction. Hence $K$ is finite. 
We come now to the main Theorem of this section. Since $G$ acts on its Cayley graph $\Gamma$ by isometries, we are interested in uniform pattern-preserving groups of quasi-isometries containing $G$.

Theorem 3.9 Let $G$ be a hyperbolic Poincare duality group and $H$ an infinite quasiconvex subgroup of infinite index in $G$. Suppose $d$ is a visual metric on $\partial G$ with $\operatorname{dim}_{\text {haus }}<\operatorname{dim}_{t}+2$, where $\operatorname{dim}_{\text {haus }}$ is the Hausdorff dimension and $\operatorname{dim}_{t}$ is the topological dimension of $(\partial G, d)$. Equivalently suppose that $\operatorname{ACD}(\partial G)<\operatorname{dim}_{t}+2$ where $\operatorname{ACD}(\partial G)$ denotes the Ahlfors regular conformal dimension of $\partial G$. Let $Q$ be a group of pattern-preserving uniform quasi-isometries containing $G$. Then $G$ is of finite index in $Q$. In particular, $Q \subset \operatorname{Comm}(G)$, where $\operatorname{Comm}(G)$ denotes the abstract commensurator of $G$.

Proof Let $L$ be the limit set of $H$ and $\mathcal{L}$ be the collection of translates of $L$ under $G$. By Corollary 1.12, we can choose a finite collection $L_{1}, \ldots, L_{n}$ of elements of $\mathcal{L}$ such that $Q_{0}=\bigcap_{i=1, \ldots, n} \operatorname{Stab}\left(L_{i}\right)$ is compact, where $\operatorname{Stab}\left(L_{i}\right)$ denotes the stabilizer of $L_{i}$ in $Q$. Then $Q_{0}$ is finite by Proposition 3.8. As in the proof of Corollary 1.13, we can choose a neighborhood $U$ of the identity in $Q$ such that $U \subset Q_{0}$. Hence $U$ is finite and $Q$ is discrete.

Let $G q_{1}, \ldots, G q_{n}, \ldots$ be distinct cosets. Since $G$ acts transitively on (the vertex set of) $\Gamma$, we can choose representatives $g_{1} q_{1}, \ldots, g_{n} q_{n}, \ldots$ such that $g_{i} q_{i}(1)=1$ for all $i$. Since (the vertex set of) $\Gamma$ is locally finite, the sequence $g_{1} q_{1}, \ldots g_{n} q_{n}, \ldots$ must have a convergent subsequence in $Q$. Since $Q$ is discrete, it follows that such a sequence must be finite. Hence $G$ is of finite index in $Q$.

Let $q \in Q$. Since $G$ is of finite index in $Q$, it follows that $q G q^{-1}$ is of finite index in $q Q q^{-1}=Q$. Therefore $G \cap q G q^{-1}$ is of finite index in $Q$. Hence $G \cap q G q^{-1}$ is of finite index in both $G$ and $q G q^{-1}$, ie $q \in \operatorname{Comm}(G)$, where $\operatorname{Comm}(G)$ denotes the abstract commensurator of $G$ (where we identify $q$ with the element of $\operatorname{Comm}(G)$ that takes $G \cap q G q^{-1}$ to $\left.q\left(G \cap q G q^{-1}\right) q^{-1}\right)$. Since distinct elements $q_{1}, q_{2}$ induce distinct homeomorphisms of $\partial G$ by definition, and since two elements defining the same element of $\operatorname{Comm}(G)$ induce the same homeomorphism on $\partial G$, the elements $q_{1}, q_{2} \in \operatorname{Comm}(G)$ are distinct. This proves the result.

In fact the proof of Theorem 3.9 gives:

Corollary 3.10 Let $G$ be a hyperbolic Poincare duality group and $H$ an infinite quasiconvex subgroup of infinite index in $G$. Suppose $d$ is a visual metric on $\partial G$ with $\operatorname{dim}_{\text {haus }}<\operatorname{dim}_{t}+2$, where $\operatorname{dim}_{\text {haus }}$ is the Hausdorff dimension and $\operatorname{dim}_{t}$ is the 
topological dimension of $(\partial G, d)$. Equivalently suppose that $\operatorname{ACD}(\partial G)<\operatorname{dim}_{t}+2$ where $\operatorname{ACD}(\partial G)$ denotes the Ahlfors regular conformal dimension of $\partial G$. Let $Q$ be a locally compact group of pattern-preserving quasi-isometries containing $G \subset$ $\operatorname{Homeo}(\partial G)$, where $Q$ is equipped with the uniform topology. Then $G$ is of finite index in $Q$. In particular, $Q \subset \operatorname{Comm}(G)$, where $\operatorname{Comm}(G)$ denotes the abstract commensurator of $G$.

Proof By Proposition 3.5, $Q$ is totally disconnected. Hence by Theorem $2.17 Q$ contains arbitrarily small compact open subgroups. Such compact open subgroups are finite by Proposition 3.8 (as in the proof of Theorem 3.9). Hence $Q$ is discrete. The rest of the proof is as in Theorem 3.9.

In the present context, Scholium 1.2 translates to the following precise statement as a consequence of Corollary 3.10.

Corollary 3.11 Let $\phi$ be a pattern-preserving quasi-isometry between pairs $\left(G_{1}, H_{1}\right)$ and $\left(G_{2}, H_{2}\right)$ of hyperbolic PD groups and infinite quasiconvex subgroups of infinite index. Suppose $d$ is a visual metric on $\partial G_{1}$ with $\operatorname{dim}_{\text {haus }}<\operatorname{dim}_{t}+2$, where $\operatorname{dim}_{\text {haus }}$ is the Hausdorff dimension and $\operatorname{dim}_{t}$ is the topological dimension of $\left(\partial G_{1}, d\right)$. Equivalently suppose that $\operatorname{ACD}\left(\partial G_{1}\right)<\operatorname{dim}_{t}+2$ where $\operatorname{ACD}\left(\partial G_{1}\right)$ denotes the Ahlfors regular conformal dimension of $\left(\partial G_{1}\right)$. Further, suppose that $G_{1}$ and $\partial \phi^{-1} \circ G_{2} \circ \partial \phi$ embed in some locally compact subgroup $Q$ of $\operatorname{Homeo}\left(\partial G_{1}\right)$ with the uniform topology. Then $G_{1}$ and $G_{2}$ are commensurable.

Proof By Corollary 3.10, $G_{1}$ is of finite index in $Q$. Hence $Q$ is a rational $\operatorname{PD}(n)$ group. Since $\partial \phi^{-1} \circ G_{2} \circ \partial \phi$ embeds in $Q$ and is also a $\operatorname{PD}(n)$ group, it follows that $\partial \phi^{-1} \circ G_{2} \circ \partial \phi \subset Q$ is of finite index [17]. Hence $G_{1}$ and $G_{2}$ are commensurable.

\section{Filling codimension one subgroups and pattern rigidity}

\subsection{Codimension one subgroups and pseudometrics}

Let $G$ be a one-ended Gromov-hyperbolic group with Cayley graph $\Gamma$. Let $H$ be a quasiconvex subgroup. We say that $H$ is codimension one if the limit set $L_{H}$ of $H$ disconnects $\partial G$. This is equivalent to saying that the join $J\left(L_{H}\right)=J$ disconnects $\Gamma$ coarsely, ie if $D$ be the quasiconvexity constant of $J$, then $\Gamma \backslash N_{D}(J)$ has more than one unbounded component, where $N_{D}(J)$ denotes the $D$-neighborhood of $J$. (See [58, Chapter 2], particularly Remark 2.4 for a proof of this equivalence and related results.) 
We say further that $H$ (or more generally a finite collection $H_{1}, \ldots, H_{k}$ ) is filling if for any two $x, y \in \partial G$, there exists a translate $g L_{H}$ of $L_{H}$ (or more generally $g L_{H_{i}}$ ) by an element $g$ of $G$ such that $x, y$ lie in distinct components of $\partial G \backslash g L_{H}$ (or more generally $\partial G \backslash g L_{H_{i}}$ ). We shall deal with a single filling subgroup for convenience of exposition. The results in this section go through for a finite collection of filling codimension one subgroups.

The existence of a finite collection of filling codimension one quasiconvex subgroups is important in light of the following Theorem due to Sageev [55] and Bergeron and Wise [2].

Theorem $4.1 \quad$ [55;2] A hyperbolic group acts properly, cocompactly on a CAT(0) cube complex if and only if it admits a finite collection $H_{1}, \ldots, H_{k}$ of filling codimension one quasiconvex subgroups.

Let $D_{1}$ be such that any path joining points in distinct unbounded components of $\Gamma \backslash N_{D}(J)$ passes within $D_{1}$ of $J$. We say that $x, y \in \Gamma$ are separated by some translate $g J$ of $J$ if $x, y$ lie in distinct unbounded components of $\Gamma \backslash g N_{D+D_{1}}(J)$. Equivalently, we shall say that the geodesic $[x, y]$ is separated by some translate $g J$ of $J$.

Lemma 4.2 Let $H$ be a codimension one, filling, quasiconvex subgroup of a oneended hyperbolic group $G$. Let $\Gamma$ be a Cayley graph of $G$. There exists $C \geq 0$ such that any geodesic $\sigma$ in $\Gamma$ of length greater than $C$ is separated by a translate of $J$.

Proof Suppose not. Then there exists a sequence of geodesic segments $\sigma_{i}=\left[a_{i}, b_{i}\right]$ which are not separated by any translate of $J$ such that $d\left(a_{i}, b_{i}\right) \rightarrow \infty$. By equivariance, we may assume that $\sigma_{i}$ is centered at the origin, ie $d\left(a_{i}, 1\right) \rightarrow \infty$ and $d\left(1, b_{i}\right) \rightarrow \infty$ and $1 \in\left[a_{i}, b_{i}\right]$. Let $a_{i} \rightarrow a_{\infty} \in \partial G$ and $b_{i} \rightarrow b_{\infty} \in \partial G$. Then $a_{\infty}$ and $b_{\infty}$ cannot lie in distinct components of $\partial G \backslash g L_{H}$ for any $g \in G$, for if they did then there exists $g \in G$ such that $a_{\infty}$ and $b_{\infty}$ lie in distinct components of $\partial G \backslash g L_{H}$ and hence for all $i$ sufficiently large, $a_{i}, b_{i}$ would lie in distinct unbounded components of $\Gamma \backslash g N_{D}(J)$. (Here we are implicitly using the correspondence between the unbounded components of $\Gamma \backslash g N_{D}(J)$ and the components of $\partial G \backslash g L_{H}$ mentioned in the first paragraph of this section; cf [58, Chapter 2]).

But if $a_{\infty}$ and $b_{\infty}$ cannot be separated, then $H$ cannot be filling, contradicting the hypothesis.

Lemma 4.3 Let $G, H, \Gamma, J$ be as above. Let $[a, b] \subset \Gamma$ be a geodesic and $c \in[a, b]$ such that $d(a, c) \geq 2 D, d(b, c) \geq 2 D$, where $D$ is the quasiconvexity constant of $J$. Suppose $g J$ separates $a, c$. Then $g J$ separates $a, b$. 
Proof Suppose not. Then $a, b$ lie in the same unbounded component of $\Gamma \backslash g N_{D}(J)$, whereas $c$ lies in a different unbounded component of $\Gamma \backslash g N_{D}(J)$. Hence there is a subsegment $[e c f]$ of $[a, b]$ such that $e, f \in N_{D_{1}} J$, but $c \notin N_{D+D_{1}} J$, contradicting the quasiconvexity constant for $J$.

Lemma 4.4 [28] Let $G$ be a hyperbolic group and $H$ a quasiconvex subgroup, with limit set $L$. Let $J$ denote the join of the limit set. There exists $N \in \mathbb{N}$ such that there exist at most $N$ distinct translates of $J$ intersecting the 2-neighborhood $B_{2}(g)$ nontrivially for any $g \in G$.

Lemma 4.5 Define a new pseudometric $\rho_{1}$ on $\Gamma$ by declaring $\rho_{1}(a, b)$ to be the number of copies of joins $J \in \mathcal{J}$ separating $a, b$. Then $\left(\Gamma, \rho_{1}\right)$ is quasi-isometric to $(\Gamma, d)$.

Proof By Lemma 4.4, it follows that there exists $N \in \mathbb{N}$ such that $d(a, b) \leq C_{0}$ implies $\rho(a, b) \leq N C_{0}$. From Lemma 4.2, it follows that there exists $C_{2} \geq 0$, such that $d(a, b) \geq C_{2}$ implies $\left.\rho(a, b)\right) \geq 1$. Now from Lemma 4.3, it follows that for $n \in \mathbb{N}$, $d(a, b) \geq n C_{2}$ implies $\left.\rho(a, b)\right) \geq n$. Hence the Lemma.

A purely topological version of Lemma 4.5 may be obtained as follows. Let $\partial^{3} G$ denote the collection of distinct unordered triples of points on $\partial G$. Then it is well known [30; 12] that $G$ acts cocompactly on $\partial^{3} G$ with metrizable quotient. Let $\rho$ be the pseudo-metric of Observation 1.5 which asserts that $\left(\partial^{3} G, \rho\right)$ is quasi-isometric to $(\Gamma, d)$.

We say that a translate $g L \in \mathcal{L}$ separates closed subsets $A, B \subset \partial G$ if $A, B$ lie in distinct components of $\partial G \backslash g L$. Define a pseudometric $\rho_{2}$ on $\partial^{3} G$ by defining $\rho_{2}\left(\left\{a_{1}, a_{2}, a_{3}\right\},\left\{b_{1}, b_{2}, b_{3}\right\}\right)$ to be the number of copies of limit sets $g L \in \mathcal{L}$ separating $\left\{a_{1}, a_{2}, a_{3}\right\},\left\{b_{1}, b_{2}, b_{3}\right\}$. Then $\left(\partial^{3} G, \rho_{2}\right)$ is quasi-isometric to $\left(\Gamma, \rho_{1}\right)$, and hence to $(\Gamma, d)$ and $\left(\partial^{3} G, \rho\right)$. We state this as follows.

Corollary 4.6 $\left(\partial^{3} G, \rho_{2}\right),\left(\Gamma, \rho_{1}\right),(\Gamma, d)$ and $\left(\partial^{3} G, \rho\right)$ are quasi-isometric to each other.

\subsection{Pattern rigidity}

We prove the following Proposition for which $G$ may be any one-ended hyperbolic group (not necessarily PD):

Proposition 4.7 Let $G$ be a one-ended hyperbolic group and $H$ a codimension one, filling, quasiconvex subgroup. Then any pattern-preserving group $Q$ of quasi-isometries is uniform. 
Proof Assume without loss of generality that $G \subset Q$. Since $Q$ consists of patternpreserving quasi-isometries, each element of $Q$ induces a pattern-preserving homeomorphism of $\partial G$. Since any pattern-preserving homeomorphism of $\partial G$ preserves $\left(\partial^{3} G, \rho_{2}\right)$ on the nose, it follows from Corollary 4.6 that $Q$ is uniform.

Combining Proposition 4.7 with Theorem 3.9 we get the following Theorem. (Note that the only PD group that is not one-ended is $\mathbb{Z}$, in which case codimension one quasiconvex subgroups in our sense do not exist.)

Theorem 4.8 Let $G$ be a PD hyperbolic group and $H$ a codimension one, filling, quasiconvex subgroup. Let $Q$ be any pattern-preserving group of quasi-isometries containing $G$. Suppose $d$ is a visual metric on $\partial G$ with $\operatorname{dim}_{\text {haus }}<\operatorname{dim}_{t}+2$, where $\operatorname{dim}_{\text {haus }}$ is the Hausdorff dimension and $\operatorname{dim}_{t}$ is the topological dimension of $(\partial G, d)$. Equivalently suppose that $\operatorname{ACD}(\partial G)<\operatorname{dim}_{t}+2$ where $\operatorname{ACD}(\partial G)$ denotes the Ahlfors regular conformal dimension of $\partial G$. Then the index of $G$ in $Q$ is finite.

In fact more is true. Combining Proposition 4.7 with Corollary 4.6, we get:

Proposition 4.9 Let $G$ be a one-ended hyperbolic group and $H$ a codimension one, filling, quasiconvex subgroup with limit set $L$. Let $\mathcal{L}$ be the collection of translates of $L$ under $G$. Then any pattern-preserving group $Q$ of homeomorphisms of $\partial G$ preserving $\mathcal{L}$ can be realized as the boundary values of uniform quasi-isometries.

Note that in Proposition 4.9 we do not need $G$ to be a PD group. Combining Proposition 4.9 with Theorem 3.9 we finally get:

Theorem 4.10 (Topological Pattern Rigidity) Let $G$ be a PD hyperbolic group and $H$ a codimension one, filling, quasiconvex subgroup with limit set $L$. Let $\mathcal{L}$ be the collection of translates of $L$ under $G$. Suppose $d$ is a visual metric on $\partial G$ with $\operatorname{dim}_{\text {haus }}<\operatorname{dim}_{t}+2$, where $\operatorname{dim}_{\text {haus }}$ is the Hausdorff dimension and $\operatorname{dim}_{t}$ is the topological dimension of $(\partial G, d)$. Equivalently suppose that $\operatorname{ACD}(\partial G)<\operatorname{dim}_{t}+2$ where $\operatorname{ACD}(\partial G)$ denotes the Ahlfors regular conformal dimension of $\partial G$. Let $Q$ be any pattern-preserving group of homeomorphisms of $\partial G$ preserving $\mathcal{L}$ and containing $G$. Then the index of $G$ in $Q$ is finite.

Theorem 4.10 is a generalization of a Theorem of Casson and Bleiler [19] and Kapovich and Kleiner [39] to all dimensions. Casson and Bleiler [19] and Kapovich and Kleiner [39] proved Theorem 4.10 for $G$ the fundamental group of a surface and $H$ an infinite cyclic subgroup corresponding to a filling curve. 


\section{Finite volume manifolds of negative curvature}

Let $M=M^{n}$ be a complete finite volume noncompact manifold of pinched negative curvature (ie $-1 \leq \chi \leq-K$ for some $K \geq 1$, where $\chi$ denotes sectional curvature). Then $\widetilde{M}$ is homeomorphic to $\mathbb{R}^{n}$ by the Cartan-Hadamard Theorem and its ideal boundary $\partial \widetilde{M}$ is homeomorphic to $S^{n-1}$. Further, by Theorem $2.12 X=\partial \widetilde{M}$ equipped with a visual metric has the structure of an Ahlfors regular metric measure space. In fact, if $-1 \leq \chi \leq-(1+\epsilon)^{2}$, the Hausdorff dimension of the visual boundary is bounded above by $(n-1)(1+\epsilon)$ (see Remark 6.1 below).

\subsection{Symmetric patterns of horoballs}

The manifold $M$ has a finite number of cusps. Lifting these to $\widetilde{M}$ we obtain an equivariant collection $c \mathcal{H}$ of horoballs. For convenience of exposition we assume that $M$ has one cusp. We shall denote individual elements of $c \mathcal{H}$ by $c H$ or $c H_{i}$. The boundary of the horoball $c H$ is called a horosphere and is denoted as $H$. The collection of horospheres will be denoted by $\mathcal{H}$. Let $G=\pi_{1}(M)$ and let $K$ denote the fundamental group of the cusp.

The collection $c \mathcal{H}$ will be called a symmetric pattern (of horoballs). It is a fact that elements of $c \mathcal{H}$ are uniformly quasiconvex (see Farb [26]) and that for any two distinct $c H_{1}, c H_{2} \in c \mathcal{H}$, there is a coarsely well-defined "centroid", ie the shortest geodesic joining $c H_{1}, c H_{2} \in c \mathcal{H}$ is coarsely well-defined (any two such lie in a uniformly bounded neighborhood of each other [26]) and hence its mid-point (the centroid of $\left.\mathrm{cH}_{1}, \mathrm{cH}_{2}\right)$ is coarsely well-defined.

In [26], Farb proves that $G$ is strongly hyperbolic relative to $K$. This is equivalent to the statement that $\widetilde{M}$ is strongly hyperbolic relative to $c \mathcal{H}$. Equivalently, the "neutered space" $\widetilde{M} \backslash \bigcup_{c H \in c \mathcal{H}} \operatorname{Int}(c H)$ is hyperbolic relative to the collection $\mathcal{H}$. We refer to [26] for background on relative hyperbolicity.

We now recast the relevant definitions and propositions of Sections 1 and 3 in the present context. Let $\Gamma, \Gamma_{K}, \Gamma_{\mathcal{K}}$ denote respectively the Cayley graph of $G$, some translate of the Cayley (sub)graph of $K$ and the collection of translates of $\Gamma_{K}$ (assuming as usual that the finite generating set of $K$ used in constructing $\Gamma_{K}$ is contained in the finite generating set of $G$ used in constructing $\Gamma$ ).

Definition 5.1 The group $\operatorname{PP}(G, K)$ of pattern-preserving maps for a (strongly) relatively hyperbolic pair $(G, K)$ as above is defined as the group of homeomorphisms of $X=\partial \widetilde{M}$ preserving (as a set) the collection of basepoints of $c \mathcal{H}$. The group $\operatorname{PPQI}(G, K)$ of pattern-preserving quasi-isometries for such a (strongly) relatively 
hyperbolic pair $(G, K)$ is defined as the subgroup of $\operatorname{PP}(G, K)$ consisting of homeomorphisms $h$ of $\partial G$ such that $h=\partial \phi$ for some quasi-isometry $\phi: \widetilde{M} \rightarrow \widetilde{M}$ that permutes the collection of horoballs $c \mathcal{H}$.

The following Theorem is a special case of a Theorem which we proved in [44] using the notion of mutual coboundedness.

Theorem 5.2 [44] Let $M=M^{n}$ be a complete finite volume manifold of pinched negative curvature and let $c \mathcal{H}$ denote the associated symmetric pattern of horoballs.

There exist two elements $\mathrm{cH}_{1}, \mathrm{cH}_{2}$ of $c \mathcal{H}$ such that the following holds.

For any $K, \epsilon$, there exists a $C$ such that if $\phi: \widetilde{M} \rightarrow \widetilde{M}$ is a pattern-preserving $(K, \epsilon)-$ quasi-isometry with $\partial \phi\left(\partial c H_{i}\right)=\partial c H_{i}$ for $i=1,2$, then $d(\phi(1), 1) \leq C$.

Let $\bar{M}=\widetilde{M} \cup \partial \widetilde{M}$ denote the Gromov compactification of $\widetilde{M}$ and $\overline{c \mathcal{H}}$ denote the collection of compactified horoballs, ie horoballs with basepoints adjoined. Let $d_{c}$ denote a metric giving the topology on $\bar{M}$. In this context Proposition 1.10 translates to the following (see [44] for instance).

Proposition 5.3 The collection $\overline{c \mathcal{H}}$ is discrete in the Hausdorff topology on the space of closed subsets of $\bar{M}$, ie for all $c H \in c \mathcal{H}$, there exists $\epsilon>0$ such that $N_{\epsilon}(c H) \cap c \mathcal{H}=c H$, where $N_{\epsilon}(c H)$ denotes an $\epsilon$ neighborhood of $c H$ in the Hausdorff metric arising from $d_{c}$.

Let $Q \subset \operatorname{PPQI}(G, K)$ be a group of quasi-isometries preserving a symmetric pattern of horoballs. Using Theorem 5.2 and Proposition 5.3, we have as in Section 3 (cf Propositions 3.3, 3.5, 3.7, 3.8):

Proposition 5.4 The group $Q$ has no nontrivial topologically infinitely divisible elements. More generally, $Q$ does not contain any nontrivial infinitely divisible subgroups. Hence $Q$ is totally disconnected. Suppose further that $\partial \widetilde{M}$ has a visual metric $d$ with $\operatorname{dim}_{\text {haus }}<\operatorname{dim}_{t}+2$, where $\operatorname{dim}_{\text {haus }}$ is the Hausdorff dimension and $\operatorname{dim}_{t}$ is the topological dimension of $(\partial \widetilde{M}, d)$. Equivalently suppose that $\operatorname{ACD}(\partial(\widetilde{M}))<\operatorname{dim}_{t}+2$ where $\operatorname{ACD}(\partial(\widetilde{M}))$ denotes the Ahlfors regular conformal dimension of $\partial(\widetilde{M})$. If $Q$ is compact, then $Q$ is finite.

Sketch of proof Proposition 3.3 and Proposition 3.5 apply directly to show that $Q$ does not have nontrivial topologically infinitely divisible elements.

Theorem 1.17 shows that $Q$ does not have arbitrarily small torsion elements. 
The rest of the argument is as in Proposition 3.8. The only point that needs to be mentioned is that $\partial^{3} \widetilde{M}$ minus a neighborhood of the cusps, rather than $\partial^{3} \widetilde{M}$ itself is quasi-isometric to the Cayley graph of $\pi_{1}(M)$. Since the quasi-isometries in $Q$ preserve the horoballs they naturally induce homeomorphisms of $\partial^{3} \widetilde{M}$ preserving horoballs. Observation 1.5 therefore goes through with the above modification.

Now, let $Q_{u} \subset \operatorname{PPQI}(G, K)$ be a group of uniform quasi-isometries preserving a symmetric pattern of horoballs. Then $Q_{u}$ is locally compact (by Lemma 1.4) and contains a compact open subgroup $K_{0}$. Suppose further that $d$ is a visual metric on $\partial(\widetilde{M})$ with $\operatorname{dim}_{\text {haus }}<\operatorname{dim}_{t}+2$, where $\operatorname{dim}_{\text {haus }}$ is the Hausdorff dimension and $\operatorname{dim}_{t}$ is the topological dimension of $(\partial(\widetilde{M}), d)$. Equivalently suppose that $\operatorname{ACD}(\partial(\widetilde{M}))<\operatorname{dim}_{t}+2$ where $\operatorname{ACD}(\partial(\widetilde{M}))$ denotes the Ahlfors regular conformal dimension of $\partial(\widetilde{M})$. Then $K_{0}$ is finite by Proposition 5.4. Hence $Q_{u}$ is discrete. Thus as in Theorem 3.9 we get the following.

Theorem 5.5 Let $M=M^{n}$ be a complete finite volume manifold of pinched negative curvature and let $c \mathcal{H}$ denote the associated symmetric pattern of horoballs. Suppose further that $d$ is a visual metric on $\partial(\widetilde{M})$ with $\operatorname{dim}_{\text {haus }}<\operatorname{dim}_{t}+2$, where $\operatorname{dim}_{\text {haus }}$ is the Hausdorff dimension and $\operatorname{dim}_{t}$ is the topological dimension of $(\partial(\widetilde{M}), d)$. Equivalently suppose that $\operatorname{ACD}(\partial(\widetilde{M}))<\operatorname{dim}_{t}+2$ where $\operatorname{ACD}(\partial(\widetilde{M}))$ denotes the Ahlfors regular conformal dimension of $\partial(\widetilde{M})$. Let $Q$ be a group of uniform quasiisometries containing $G$ preserving a symmetric pattern of horoballs. Then $G$ is of finite index in $Q$. In particular, $Q \subset \operatorname{Comm}(G)$, where $\operatorname{Comm}(G)$ denotes the abstract commensurator of $G$.

\subsection{Weak QI rigidity for relatively hyperbolic groups}

We shall be using the following Theorem of Behrstock, Druţu and Mosher (which follows from the proof of [1, Theorem 4.8]; see also Schwartz [56]) and Farb's result [26] that the fundamental group of complete finite volume noncompact manifold of pinched negative curvature is strongly hyperbolic relative to the cusp groups.

Theorem 5.6 (Behrstock-Druţu-Mosher [1], Schwartz [56]) Let $M=M^{n}$ be a complete finite volume manifold of pinched negative curvature with $n>2$ (and one cusp for ease of exposition). Let $G=\pi_{1}(M)$. Let $K$ denote the fundamental group of the cusp. Choose a finite generating set for $G$ containing a finite generating set for $K$. Let $\Gamma, \Gamma_{K}, \Gamma_{\mathcal{K}}$ denote respectively the Cayley graph of $G$, some translate of the Cayley (sub)graph of $K$ and the collection of translates of $\Gamma_{K}$. Then for every $L \geq 1$ and $C \geq 0$ there exists $R=R(L, C, G, K)$ such that the following holds.

For any $(L, C)$-(self-)quasi-isometry $q$ of $G$, the image $q\left(\Gamma_{K}\right)$ is at a bounded Hausdorff distance $R$ of some $g \Gamma_{K} \in \Gamma_{\mathcal{K}}$. 
Let $q$ be a (self-)quasi-isometry of $\Gamma$. Elements of the collection $\Gamma_{\mathcal{K}}$ are mapped bijectively to bounded neighborhoods of elements of collection $\Gamma_{\mathcal{K}}$. Identifying $\Gamma$ with the neutered space $\left(\widetilde{M} \backslash \bigcup_{c H \in c \mathcal{H}} \operatorname{Int}(c H)\right)$ we can extend $q$ (cf [56]) to a (self-)quasiisometry $q^{h}$ of $\widetilde{M}$ where the elements of $c \mathcal{H}$ are bijectively mapped to uniformly bounded neighborhoods of elements of $c \mathcal{H}$. Each element of $c \mathcal{H}$ has a unique limit point in $\partial \widetilde{M}$ which we shall call its basepoint. Let $\partial q$ denote the induced map of $\partial(\widetilde{M})$ and $\partial \mathcal{H}$ denote the collection of basepoints of $c \mathcal{H}$ in $\partial \widetilde{M}$. Thus a simple consequence of Theorem 5.6 is the following.

Corollary 5.7 Let $M=M^{n}$ be a complete finite volume manifold of pinched negative curvature with $n>2$ (and one cusp for ease of exposition). Let $G=\pi_{1}(M)$ ). Let $K$ denote the fundamental group of the cusp. Let $c \mathcal{H}$ be the associated symmetric pattern of horoballs in $\widetilde{M}$. Choose a finite generating set for $G$ containing a finite generating set for $K$. Let $\Gamma, \Gamma_{K}, \Gamma_{\mathcal{K}}$ denote respectively the Cayley graph of $G$, some translate of the Cayley (sub)graph of $K$ and the collection of translates of $\Gamma_{K}$. Identify $\Gamma$ (coarsely) with the neutered space $\left(\widetilde{M} \backslash \bigcup_{c H \in c \mathcal{H}} \operatorname{Int}(c H)\right)$.

Then for every $L \geq 1$ and $C \geq 0$ there exist $L_{1} \geq 1, C_{1} \geq 0$ and $R=R(L, C, G, K)$ such that the following holds.

For any $(L, C)$-(self-)quasi-isometry $q$ of $\Gamma$ (or equivalently, $\left(\widetilde{M} \backslash \bigcup_{c H \in c \mathcal{H}} \operatorname{Int}(c H)\right)$ ), there is an $\left(L_{1}, C_{1}\right)$-(self-)quasi-isometry $q^{h}$ of $\widetilde{M}$ such that the image $q^{h}\left(\Gamma_{K}\right)$ is at a bounded Hausdorff distance $R$ of some $g \Gamma_{K} \in \Gamma_{\mathcal{H}}$. Hence $q$ induces a homeomorphism $\partial q$ of $\partial(\widetilde{M})$ preserving the basepoints of $c \mathcal{H}$.

Combining Corollary 5.7 with Theorem 5.5 we get the following.

Theorem 5.8 Let $M=M^{n}$ be a complete finite volume manifold of pinched negative curvature with $n>2$. Let $G=\pi_{1}(M)$. Suppose that there exists a visual metric $d$ on $\partial(\widetilde{M})$ with $\operatorname{dim}_{\text {haus }}<\operatorname{dim}_{t}+2$, where $\operatorname{dim}_{\text {haus }}$ is the Hausdorff dimension and $\operatorname{dim}_{t}$ is the topological dimension of $(\partial(\widetilde{M}), d)$. Equivalently suppose that $\operatorname{ACD}(\partial(\widetilde{M}))<\operatorname{dim}_{t}+2$, where $\operatorname{ACD}(\partial(\widetilde{M}))$ denotes the Ahlfors regular conformal dimension of $\partial(\widetilde{M})$.

Let $\Gamma$ be a Cayley graph of $G$ with respect to a finite generating set. Let $Q$ be a group of uniform quasi-isometries of $\Gamma$ containing $G$. Then $G$ is of finite index in $Q$. In particular, $Q \subset \operatorname{Comm}(G)$, where $\operatorname{Comm}(G)$ denotes the abstract commensurator of $G$. 


\section{Examples and consequences}

\subsection{Examples}

In this subsection we list a collection of examples of hyperbolic $\operatorname{PD}(n)$ groups.

Rank one symmetric spaces Uniform lattices in rank one symmetric spaces are examples of hyperbolic $\operatorname{PD}(n)$ groups. For lattices in real hyperbolic space $\mathbf{H}^{n}$ the usual visual metric has Hausdorff dimension dim haus equal to topological dimension $\operatorname{dim}_{t}$. For lattices in complex hyperbolic space $\mathbb{C} \mathbf{H}^{n}$ the usual visual metric has dimhaus $=$ $\operatorname{dim}_{t}+1$. Lattices in quaternionic hyperbolic space and the Cayley hyperbolic plane cannot have codimension one subgroups; so Theorem 3.9 cannot apply. But these spaces are QI-rigid by a deep Theorem of Pansu [49].

Gromov-Thurston examples Gromov and Thurston [33] construct examples of closed negatively curved $n$-manifolds ( $n>3$ ) of arbitrarily pinched negative curvature, which do not admit metrics of constant negative curvature.

Remark 6.1 For these examples the inequality $\operatorname{dim}_{\text {haus }}<\operatorname{dim}_{t}+2$ is satisfied. This is because of the following. If $-1 \leq \chi \leq-(1+\epsilon)^{2}$, the rate of divergence of geodesics is bounded by $O\left(e^{(1+\epsilon) R}\right)$. Hence volumes of $R$-balls is bounded by $O\left(e^{(n-1)(1+\epsilon) R}\right)$. Therefore the Hausdorff dimension of the visual boundary is bounded above by $(n-1)(1+\epsilon)$ (see [61, Theorem 1]).

Mostow-Siu examples Mostow and Siu [47] constructed an infinite family of complex surfaces that admit negatively curved Kahler metrics but do not admit a complex hyperbolic structure. Fundamental groups of these provide further examples of hyperbolic $\mathrm{PD}(n)$ groups.

Davis-Januskiewicz examples A remarkable set of examples is constructed by Davis and Januskiewicz [24], who show that there exist hyperbolic $\operatorname{PD}(n)$ groups $G$ for $n \geq 4$, such that the boundary $\partial G$ is not homeomorphic to the sphere $S^{n-1}$. $\partial G$ need not be simply connected or locally simply connected and hence is not even an Absolute Neighborhood Retract.

\subsection{Quasi-isometric rigidity}

Let $\mathcal{A}$ be a graph of groups with Bass-Serre tree of spaces $X \rightarrow T$. Let $A=\pi_{1} \mathcal{A}$. Let $\mathcal{V E}(T)$ be the set of vertices and edges of $T$. The metric on $T$ induces a metric 
on $\mathcal{V E}(T)$, via a natural injection $\mathcal{V E}(T) \rightarrow T$ which takes each vertex to itself and each edge to its midpoint. Let $d_{H}$ denote Hausdorff distance.

We refer the reader to Mosher, Sageev and Whyte [46] specifically for the following notions:

(1) depth zero raft

(2) crossing graph condition

(3) coarse finite type and coarse dimension

(4) finite depth

Combining Theorems 1.5, 1.6 of Mosher, Sageev and Whyte [46] with the Pattern Rigidity Theorem 4.8 we have the following QI-rigidity Theorem along the lines of Theorem 7.1 of [46].

Theorem 6.2 Let $\mathcal{A}$ be a finite, irreducible graph of groups such that for the associated Bass-Serre tree $T$ of spaces,

(a) the vertex groups are $\operatorname{PD}(n)$ hyperbolic groups for some fixed $n$,

(b) edge groups are filling codimension one in the adjacent vertex groups,

and such that $\mathcal{A}$ is of finite depth. Further suppose that each vertex group $G$ admits a visual metric $d$ on $\partial G$ with $\operatorname{dim}_{\text {haus }}<\operatorname{dim}_{t}+2$, where $\operatorname{dim}_{\text {haus }}$ is the Hausdorff dimension and $\operatorname{dim}_{t}$ is the topological dimension of $(\partial G, d)$. Equivalently suppose that $\operatorname{ACD}(\partial G)<\operatorname{dim}_{t}+2$ where $\operatorname{ACD}(\partial G)$ denotes the Ahlfors regular conformal dimension of $\partial G$.

If $H$ is a finitely generated group quasi-isometric to $A=\pi_{1}(\mathcal{A})$ then $H$ splits as a graph $\mathcal{A}^{\prime}$ of groups whose depth zero vertex groups are commensurable to the depth zero vertex groups of $\mathcal{A}$ and whose edge groups and positive depth vertex groups are quasi-isometric to groups of type (b).

Proof By the restrictions on the vertex and edge groups, it automatically follows that all vertex and edge groups are PD groups of coarse finite type. Since the edge groups are filling, the crossing graph condition of Theorems 1.5, 1.6 of [46] is satisfied. $\mathcal{A}$ is automatically finite depth, because an infinite index subgroup of a $\operatorname{PD}(n)$ groups has coarse dimension at most $n-1$.

Then by [46, Theorems 1.5 and 1.6], $H$ splits as a graph of groups $\mathcal{A}^{\prime}$ with depth zero vertex spaces quasi-isometric to the vertex groups of $\mathcal{A}$ and edge groups quasi-isometric to the edge groups of $\mathcal{A}$. Further, the quasi-isometry respects the vertex and edge 
spaces of this splitting, and thus the quasi-actions of the vertex groups on the vertex spaces of $\mathcal{A}$ preserve the patterns of edge spaces.

By Theorem 4.8 the depth zero vertex groups in $\mathcal{A}^{\prime}$ are commensurable to the corresponding groups in $\mathcal{A}$.

\subsection{The permutation topology}

In this paper we have ruled out three kinds of elements from the group $\operatorname{PPQI}(G, H)$ of pattern-preserving quasi-isometries under appropriate hypotheses on $G$ :

(a) elements that admit arbitrarily small roots (topologically divisible elements)

(b) arbitrarily small torsion elements (essentially Theorem 1.17)

(c) elements with arbitrarily large powers close to the identity (no copies of the $p$-adics)

In a sense (a) and (c) are phenomena that are opposite to each other. In hindsight, the previous works on pattern rigidity $[57 ; 7 ; 6]$ exploited (a) in the context of an ambient Lie group which automatically rules out (b) and (c).

We have used the fact that the group is pattern-preserving in a rather weak sense, only to conclude that the group we are interested in is totally disconnected. In fact Theorem 3.9 generalizes readily to show that a locally compact totally disconnected group of quasiisometries containing $G$ must be a finite extension of $G$ under appropriate hypotheses on $G$. The crucial hypothesis is local compactness on $\operatorname{PPQI}(G, H)$ which can be removed under hypotheses on $H$ as in Theorem 4.8. We would like to remove the hypothesis of local compactness in more general situations.

Remark 3.6 gives a reasonably explicit structure of $K$ for $K$ a compact group of pattern-preserving quasi-isometries. $K$ acts on the discrete set $\mathcal{L}$ of patterns with finite orbits $\mathcal{L}_{1}, \mathcal{L}_{2}, \ldots$ and hence $K \subset \prod_{i} S\left(\mathcal{L}_{i}\right)$, where $S\left(\mathcal{L}_{i}\right)$ denotes the symmetric group on the finite set $\mathcal{L}_{i}$ and $\prod$ denotes direct product.

To establish Theorem 3.9 without the hypothesis of local compactness, two crucial problems remain:

Problem 1 A topological converse to Remark 3.6 which would say that a group $K \subset \prod_{i} S\left(\mathcal{L}_{i}\right)$ acting with finite orbits on $\mathcal{L}$ must be compact in the uniform topology on $\partial G$. 
As an approach to this, we propose an alternate topology on $\operatorname{PPQI}(G, H)$ and call it the permutation topology. Enumerate $\mathcal{L}=L_{1}, L_{2}, \ldots$. Since the representation of $\operatorname{PPQI}(G, H)$ in the symmetric group of permutations $S(\mathcal{L})$ is faithful, we declare that a system of open neighborhoods of the origin in $\operatorname{PPQI}(G, H)$ is given by the set $U_{N}$ of elements of $\operatorname{PPQI}(G, H)$ fixing $L_{i}, i=1, \ldots, N$. Now consider an element $\phi \in$ $\operatorname{PPQI}(G, H)$ acting with finite orbits on $\mathcal{L}$. Then the (closed) monothetic subgroup $(\phi)$ generated by $\phi$ is locally compact and by Corollary 2.25, under certain hypotheses, it cannot contain the $p$-adics. Hence it must have arbitrarily small torsion elements. We cannot apply Theorem 1.17 right away. To be able to apply Theorem 1.17, we need to show the following:

For $\epsilon$ as in Theorem 1.17, there exists $N$ such that for all $k$, if $\phi^{k}$ stabilizes each $L_{i}$, $i=1, \ldots, N$, then each orbit of $\phi^{k}$ has diameter less than $\epsilon$.

Thus a weak enough statement ensuring a comparison of the permutation topology with the uniform topology is necessary. The coarse barycenter construction of [44] might be helpful here to construct quasi-isometries coarsely fixing large balls and providing a starting point for the problem.

Problem 2 A more important and more difficult problem is to rule out elements of $\operatorname{PPQI}(G, H)$ which fix finitely many elements of $\mathcal{L}$ (and hence coarsely fix the origin in $G$ by Theorem 1.8) but act with at least one unbounded orbit on $\mathcal{L}$. We would have to show the following:

There exists $N$ such that if $\phi \in \operatorname{PPQI}(G, H)$ stabilizes each $L_{i}, i=1, \ldots, N$, then each orbit of $\phi$ is finite.

Acknowledgements I would like to thank Mladen Bestvina, Kingshook Biswas, Marc Bourdon, Emmanuel Breuillard and George Willis for helpful conversations and correspondence. Special thanks are due to Misha Gromov for an extremely instructive conversation during which he told me about Scholium 1.2.

This work was done in part while the author was visiting Caroline Series at the Mathematics Institute, University of Warwick and the Universite Paris-Sud, Orsay under the Indo-French ARCUS programme. I thank these institutions for their hospitality.

This research was partly supported by CEFIPRA research project grant 4301-1.

\section{References}

[1] J Behrstock, C Druţu, L Mosher, Thick metric spaces, relative hyperbolicity, and quasi-isometric rigidity, Math. Ann. 344 (2009) 543-595 MR2501302 
[2] N Bergeron, D Wise, A boundary criterion for cubulation arXiv:0908.3609

[3] M Bestvina, Local homology properties of boundaries of groups, Michigan Math. J. 43 (1996) 123-139 MR1381603

[4] M Bestvina, G Mess, The boundary of negatively curved groups, J. Amer. Math. Soc. 4 (1991) 469-481 MR1096169

[5] C J Bishop, P W Jones, Hausdorff dimension and Kleinian groups, Acta Math. 179 (1997) 1-39 MR1484767

[6] K Biswas, Flows, fixed points and rigidity in Kleinian groups, to appear in Geom. Funct. Anal. arXiv:0903.2419

[7] K Biswas, M Mj, Pattern rigidity in hyperbolic spaces: duality and PD subgroups, Groups Geom. Dyn. 6 (2012) 97-123

[8] M Bonk, B Kleiner, Quasisymmetric parametrizations of two-dimensional metric spheres, Invent. Math. 150 (2002) 127-183 MR1930885

[9] M Bonk, O Schramm, Embeddings of Gromov hyperbolic spaces, Geom. Funct. Anal. 10 (2000) 266-306 MR1771428

[10] M Bourdon, H Pajot, Cohomologie $l_{p}$ et espaces de Besov, J. Reine Angew. Math. 558 (2003) 85-108 MR1979183

[11] B H Bowditch, Cut points and canonical splittings of hyperbolic groups, Acta Math. 180 (1998) 145-186 MR1638764

[12] B H Bowditch, A topological characterisation of hyperbolic groups, J. Amer. Math. Soc. 11 (1998) 643-667 MR1602069

[13] G E Bredon, Orientation in generalized manifolds and applications to the theory of transformation groups, Michigan Math. J. 7 (1960) 35-64 MR0116342

[14] G E Bredon, Introduction to compact transformation groups, Pure and Applied Math. 46, Academic Press, New York (1972) MR0413144

[15] G E Bredon, Sheaf theory, second edition, Graduate Texts in Math. 170, Springer, New York (1997) MR1481706

[16] M R Bridson, A Haefliger, Metric spaces of non-positive curvature, Grundl. Math. Wissen. 319, Springer, Berlin (1999) MR1744486

[17] KS Brown, Cohomology of groups, Graduate Texts in Math. 87, Springer, New York (1994) MR1324339

[18] S Buyalo, V Schroeder, Elements of asymptotic geometry, EMS Monogr. in Math., European Math. Soc. (2007) MR2327160

[19] A J Casson, S A Bleiler, Automorphisms of surfaces after Nielsen and Thurston, London Math. Soc. Student Texts 9, Cambridge Univ. Press (1988) MR964685 
[20] R R Coifman, G Weiss, Analyse harmonique non-commutative sur certains espaces homogènes: Étude de certaines intégrales singulières, Lecture Notes in Math. 42, Springer, Berlin (1971) MR0499948

[21] P E Conner, E E Floyd, A characterization of generalized manifolds, Michigan Math. J. 6 (1959) 33-43 MR0102818

[22] M Coornaert, Mesures de Patterson-Sullivan sur le bord d'un espace hyperbolique au sens de Gromov, Pacific J. Math. 159 (1993) 241-270 MR1214072

[23] M W Davis, Poincaré duality groups, from: "Surveys on surgery theory, Vol. 1", (S Cappell, A Ranicki, J Rosenberg, editors), Ann. of Math. Stud. 145, Princeton Univ. Press (2000) 167-193 MR1747535

[24] M W Davis, T Januszkiewicz, Hyperbolization of polyhedra, J. Differential Geom. 34 (1991) 347-388 MR1131435

[25] K J Falconer, Dimensions and measures of quasi self-similar sets, Proc. Amer. Math. Soc. 106 (1989) 543-554 MR969315

[26] B Farb, Relatively hyperbolic groups, Geom. Funct. Anal. 8 (1998) 810-840 MR1650094

[27] É Ghys, P de la Harpe (editors), Sur les groupes hyperboliques d'après Mikhael Gromov, Progress in Math. 83, Birkhäuser, Boston (1990) MR1086648 Papers from the Swiss Seminar on Hyperbolic Groups held in Bern, 1988

[28] R Gitik, M Mitra, E Rips, M Sageev, Widths of subgroups, Trans. Amer. Math. Soc. 350 (1998) 321-329 MR1389776

[29] A M Gleason, Groups without small subgroups, Ann. of Math. 56 (1952) 193-212 MR0049203

[30] M Gromov, Hyperbolic groups, from: "Essays in group theory", (S M Gersten, editor), Math. Sci. Res. Inst. Publ. 8, Springer, New York (1987) 75-263 MR919829

[31] M Gromov, Asymptotic invariants of infinite groups, from: "Geometric group theory, Vol. 2 (Sussex, 1991)”, (G A Niblo, M A Roller, editors), London Math. Soc. Lecture Note Ser. 182, Cambridge Univ. Press (1993) 1-295 MR1253544

[32] M Gromov, personal communication (2009)

[33] M Gromov, W Thurston, Pinching constants for hyperbolic manifolds, Invent. Math. 89 (1987) 1-12 MR892185

[34] A Hatcher, Algebraic topology, Cambridge Univ. Press (2002) MR1867354

[35] J Heinonen, Lectures on analysis on metric spaces, Universitext, Springer, New York (2001) MR1800917

[36] E Hewitt, K A Ross, Abstract harmonic analysis. Vol. I: Structure of topological groups. Integration theory, group representations, Grundl. Mathl. Wissen. 115, Academic Press, New York (1963) MR0156915 
[37] J G Hocking, G S Young, Topology, Addison-Wesley, Reading, MA-London (1961) MR0125557

[38] T Iwaniec, G Martin, Geometric function theory and non-linear analysis, Oxford Math. Monogr., The Clarendon Press, Oxford Univ. Press, New York (2001) MR1859913

[39] M Kapovich, B Kleiner, Hyperbolic groups with low-dimensional boundary, Ann. Sci. École Norm. Sup. 33 (2000) 647-669 MR1834498

[40] S Lefschetz, Topics in Topology, Annals of Math. Studies 10, Princeton Univ. Press (1942) MR0007094

[41] J Luukkainen, E Saksman, Every complete doubling metric space carries a doubling measure, Proc. Amer. Math. Soc. 126 (1998) 531-534 MR1443161

[42] G J Martin, The Hilbert-Smith conjecture for quasiconformal actions, Electron. Res. Announc. Amer. Math. Soc. 5 (1999) 66-70 MR1694197

[43] J Milnor, On the Steenrod homology theory, from: "Novikov conjectures, index theorems and rigidity, Vol. 1 (Oberwolfach, 1993)", (S C Ferry, A Ranicki, J Rosenberg, editors), London Math. Soc. Lecture Note Ser. 226, Cambridge Univ. Press (1995) 79-96 MR1388297

[44] M Mj, Relative rigidity, quasiconvexity and C-complexes, Algebr. Geom. Topol. 8 (2008) 1691-1716 MR2448868

[45] D Montgomery, L Zippin, Topological transformation groups, Interscience, New York-London (1955) MR0073104

[46] L Mosher, M Sageev, K Whyte, Quasi-actions on trees II: Finite depth Bass-Serre trees, Mem. Amer. Math. Soc. 214 (2011) vi+105 MR2867450

[47] G D Mostow, Y T Siu, A compact Kähler surface of negative curvature not covered by the ball, Ann. of Math. 112 (1980) 321-360 MR592294

[48] M H A Newman, A theorem on periodic transformation of spaces, Quart. J. Math. Oxford Ser. (2) 2 (1931) 1-8

[49] P Pansu, Métriques de Carnot-Carathéodory et quasiisométries des espaces symétriques de rang un, Ann. of Math. 129 (1989) 1-60 MR979599

[50] F Paulin, Un groupe hyperbolique est déterminé par son bord, J. London Math. Soc. 54 (1996) 50-74 MR1395067

[51] F Paulin, On the critical exponent of a discrete group of hyperbolic isometries, Differential Geom. Appl. 7 (1997) 231-236 MR1480536

[52] F Raymond, $R L$ Wilder's work on generalized manifolds-an appreciation, from: "Algebraic and geometric topology (Proc. Sympos., Univ. California, Santa Barbara, CA, 1977)”, (K C Millett, editor), Lecture Notes in Math. 664, Springer, Berlin (1978) 7-32 MR518402 
[53] F Raymond, R F Williams, Examples of p-adic transformation groups, Ann. of Math. 78 (1963) 92-106 MR0150769

[54] D Repovš, E S̆čepin, A proof of the Hilbert-Smith conjecture for actions by Lipschitz maps, Math. Ann. 308 (1997) 361-364 MR1464908

[55] M Sageev, Ends of group pairs and non-positively curved cube complexes, Proc. London Math. Soc. 71 (1995) 585-617 MR1347406

[56] R E Schwartz, The quasi-isometry classification of rank one lattices, Inst. Hautes Études Sci. Publ. Math. (1995) 133-168 MR1383215

[57] R E Schwartz, Symmetric patterns of geodesics and automorphisms of surface groups, Invent. Math. 128 (1997) 177-199 MR1437498

[58] P Scott, G A Swarup, Regular neighbourhoods and canonical decompositions for groups, Astérisque 289, Soc. Math. France (2003) MR2032389

[59] P A Smith, Transformations of finite period. III: Newman's theorem, Ann. of Math. 42 (1941) 446-458 MR0004128

[60] D Sullivan, The density at infinity of a discrete group of hyperbolic motions, Inst. Hautes Études Sci. Publ. Math. (1979) 171-202 MR556586

[61] D Sullivan, Entropy, Hausdorff measures old and new, and limit sets of geometrically finite Kleinian groups, Acta Math. 153 (1984) 259-277 MR766265

[62] G A Swarup, On the cut point conjecture, Electron. Res. Announc. Amer. Math. Soc. 2 (1996) 98-100 MR1412948

[63] E L Swenson, On Axiom H, Michigan Math. J. 46 (1999) 3-11 MR1682883

[64] J Väisälä, Gromov hyperbolic spaces, Expo. Math. 23 (2005) 187-231 MR2164775

[65] H Yamabe, On the conjecture of Iwasawa and Gleason, Ann. of Math. 58 (1953) 48-54 MR0054613

[66] C-T Yang, Transformation groups on a homological manifold, Trans. Amer. Math. Soc. 87 (1958) 261-283 MR0100271

[67] C-T Yang, p-adic transformation groups, Michigan Math. J. 7 (1960) 201-218 MR0120310

School of Mathematical Sciences, RKM Vivekananda University

PO Belur Math, Dt Howrah WB-711202, India

mahan@rkmvu.ac.in

http://maths.rkmvu.ac.in/ mahan/

Proposed: Benson Farb

Received: 9 January 2010

Seconded: David Gabai, Ronald Fintushel

Revised: 24 March 2012 